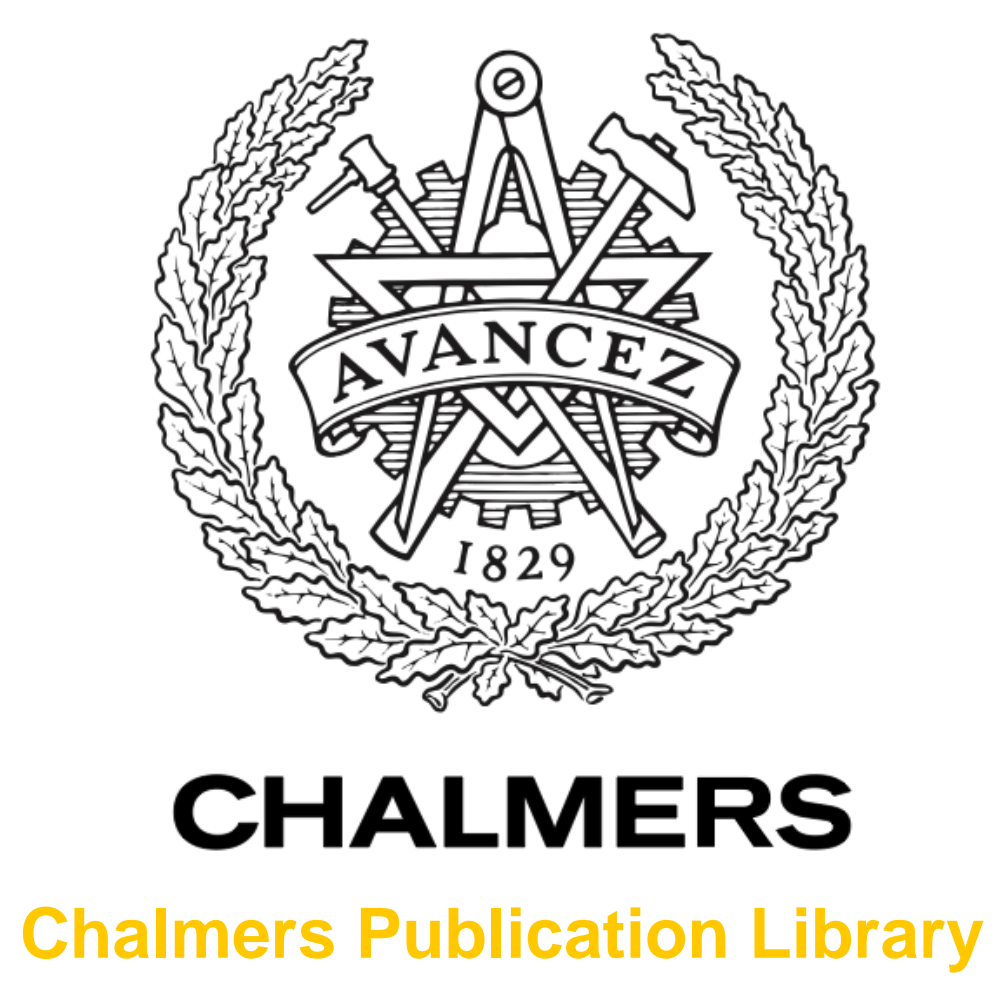

\title{
Electromobility Studies Based on Convex Optimization DESIGN AND CONTROL ISSUES REGARDING VEHICLE ELECTRIFICATION
}

This document has been downloaded from Chalmers Publication Library (CPL). It is the author's version of a work that was accepted for publication in:

IEEE Control Systems (ISSN: 1066-033X)

Citation for the published paper:

Egardt, B. ; Murgovski, N. ; Pourabdollah, M. et al. (2014) "Electromobility Studies Based on Convex Optimization DESIGN AND CONTROL ISSUES REGARDING VEHICLE

ELECTRIFICATION". IEEE Control Systems, vol. 34(2),

http://dx.doi.org/10.1109/MCS.2013.2295709

Downloaded from: http://publications.lib.chalmers.se/publication/193199

Notice: Changes introduced as a result of publishing processes such as copy-editing and formatting may not be reflected in this document. For a definitive version of this work, please refer to the published source. Please note that access to the published version might require a subscription.

Chalmers Publication Library (CPL) offers the possibility of retrieving research publications produced at Chalmers University of Technology. It covers all types of publications: articles, dissertations, licentiate theses, masters theses, conference papers, reports etc. Since 2006 it is the official tool for Chalmers official publication statistics. To ensure that Chalmers research results are disseminated as widely as possible, an Open Access Policy has been adopted.

The CPL service is administrated and maintained by Chalmers Library. 


\title{
Electromobility studies based on convex optimization
}

\author{
Bo Egardt, Nikolce Murgovski, Mitra Pourabdollah, Lars Johannesson
}

November 29, 2013

The electrification of road transport is accelerating globally, propelled by a mix of environmental concerns, legislative mandates, and business opportunities. Relying to a larger extent on electricity in the transportation sector provides new opportunities to reduce $\mathrm{CO}_{2}$ emissions, fossil fuel consumption, and local air pollution by improving energy efficiency and employing renewable energy. As part of this development, leading vehicle manufacturers are currently making a substantial effort to provide Hybrid Electric Vehicles (HEVs), Plug-in Hybrid Electric Vehicles (PHEVs), and pure Electric Vehicles (EVs) to the market.

This potentially major change in the transportation system entails significant challenges, [1], [2]. The technology in several vital areas, such as components for electric energy storage and electric drives, needs to be developed further. Effective control design tools are also needed to master the increased complexity of the new powertrains. This increased complexity concerns both design and operation of vehicles, and the interplay between vehicles and the infrastructure, such as the electric grid and systems for navigation and traffic information.

There are several interesting control design issues related to vehicle electrification. In this article, we will focus on some of these issues for two common powertrain configurations, known as series and parallel, respectively; see "Hybrid Electric Powertrains" for an introductory description. The two configurations share a common characteristic, namely the additional degree of freedom compared to a conventional powertrain. In response to the driver's power demand, as expressed by the accelerator command, there is a need for the control system to arbitrate 
between the two power sources; in the parallel configuration the combustion engine and the electric machine, in the series configuration the engine/generator unit and the electric storage system. This control task, which can also be seen as the task to control the energy buffer, most often a battery, is referred to as energy management. The energy management problem has been investigated thoroughly and the literature on the topic is rich. A survey with many references is found in [3] and the book [4] gives an introduction to the area. The energy management problem can be approached in many different ways, but in addition to purely ad hoc solutions, some type of optimal control formulation is the dominant approach; dynamic programming (DP) and solutions based on the Pontryagin principle are often used. The basic formulations are based on exact knowledge of the driving mission, and the solutions provide the optimal energy management over the defined mission. The ideal solutions are used for benchmarking and can also be used to find approximate, sub-optimal control strategies for on-line use in the vehicle. One of the most used strategies, Equivalent Consumption Minimization Strategy (ECMS), can be seen as an approximation of an optimal control formulation [4].

In addition to the energy management problem, an important design task is related to the sizing of driveline components. This statement applies to conventional drivelines as well, but the increased complexity of an electrified powertrain makes design of the powertrain a challenging task. One reason is that the battery is a major bottleneck in the electrified powertrain and needs to be sized with care. Indeed, many studies have been performed on how to optimally size the driveline components, in particular the battery. These studies rely on assessing both component costs and energy (fuel and electricity) consumption over a collection of driving missions. An optimization algorithm is used to find the best design compromise in terms of component sizing/scaling parameters. Examples of such studies are found in [5], [6], [7].

The two basic design tasks mentioned above, the energy management and the component sizing, are actually strongly coupled. The energy management strategy depends on the component properties, and the optimal sizing depends on how the energy management works. The latter problem is often handled by decoupling the plant and controller, and then optimizing them sequentially or iteratively, [5], [6], [7], [8], [9]. Sequential and iterative strategies, however, generally fail to achieve global optimality [10]. An alternative is to apply a nested plant/control 
optimization strategy [8]. The strategy comprises two nested loops: an outer loop where the system objective is optimized over the set of feasible plants and an inner loop that generates optimal controls for plants chosen by the outer loop. While this approach delivers the globally optimal solution, it either incurs a heavy computational burden (when for example dynamic programming is used to optimize the energy management) or requires substantial modeling approximations [11], [12], [13]. In contrast, the objective of this article is to present a design approach that offers the possibility to optimize simultaneously the energy management strategy and the component sizing. The key element of this approach is to find modeling approximations that allow convex optimization techniques to be applied. The intention of this work is to exploit the computational efficiency offered by convex solvers in order to facilitate studies during the early design phase. The approach can be used, for example, over very long driving missions or to perform many optimizations in comprehensive feasibility studies.

The application of convex optimization to the design and operation of electrified powertrains is not new. The potential of using convex optimization in this context was pointed out already in the late 90s in [14], and more recently [15], [16], [17], [18], [19]. In these studies, convex optimization was used to compute the optimal energy management, either over an entire driving cycle or, as an ingredient of a predictive control scheme, over a limited time horizon. The possibility to use convex optimization to combine the computation of an optimal energy management with optimal component sizing was introduced in [20], [21], where the charging infrastructure was also included into the problem.

The aim of this article is to give an overview of the approach for the combined optimal design and operation of electrified vehicles, based on convex optimization. The presentation is based on a slight reformulation of the problem, as compared with the references given above. The main purpose of this reformulation is to describe the driveline components in a unified manner in terms of energy and power variables. This approach allows a simple and accessible description of the main ideas. To illustrate how the method can be used, two case studies are briefly presented. These studies are described in more detail in the references given.

The article is organized as follows. The next section describes a design example in order to give an introduction to the type of problems addressed. The following section describes the 
convex modeling of the powertrain components, and how these models can be used to formulate and solve a convex optimization problem. To illustrate the potential of the method, two case studies are then presented. Finally, some extensions of the method are discussed and references are given for further reading.

\section{A design example: sizing the buffer of a city bus}

The task of sizing the buffer of an electrified city bus is a typical example of the type of problems adressed and will be used to introduce some of the ideas. The bus is a PHEV with a series powertrain configuration based on a combined engine-generator unit (EGU) and an electrical motor; for details, see "Hybrid Electric Powertrains". In this example, the electric buffer could be either a battery or a supercapacitor, or a combination of both.

In addition to following a time schedule, the bus must also follow a certain velocity/acceleration trajectory in order to comply with traffic limitations, driveability, and passengers' comfort requirements. Then, a bus line can be fully described by the desired velocity profile, road altitude, and information about average stand-still intervals at bus stops or traffic stops. In this case, the bus line, depicted in Figure 1, starts and ends at the same bus stop. Fast-charge docking stations are installed at seven bus stops along the bus line, and a tight duty schedule is considered that prevents charging for longer than $10 \mathrm{~s}$ while standing still at these bus stops. By design, the circular bus line conserves the vehicle's kinetic and potential energy at the beginning and the end of the line. Furthermore, to study the operational efficiency of the PHEV, there is an additional condition that the initial and final energy in the buffer should be the same.

The operational cost of this vehicle depends mainly on the quantities of diesel fuel and electricity consumed along the bus route. Then, when designing a vehicle based on minimizing the operational cost, it is beneficial to increase the usage of electric energy. The reasons for this are twofold: the electricity price per unit energy is generally lower than that of diesel fuel with the price difference expected to increase in coming years [22] and electric components typically operate with much higher efficiency than that of the internal combustion engine (ICE) [4]. However, higher utilization of electric energy requires a larger buffer, which leads to increased 


\section{TABLE I}

OPTIMIZATION PROBLEM FOR OPTIMAL DESIGN OF ELECTRIFIED VEHICLES WITH ACCESS

TO PREDICTIVE INFORMATION.

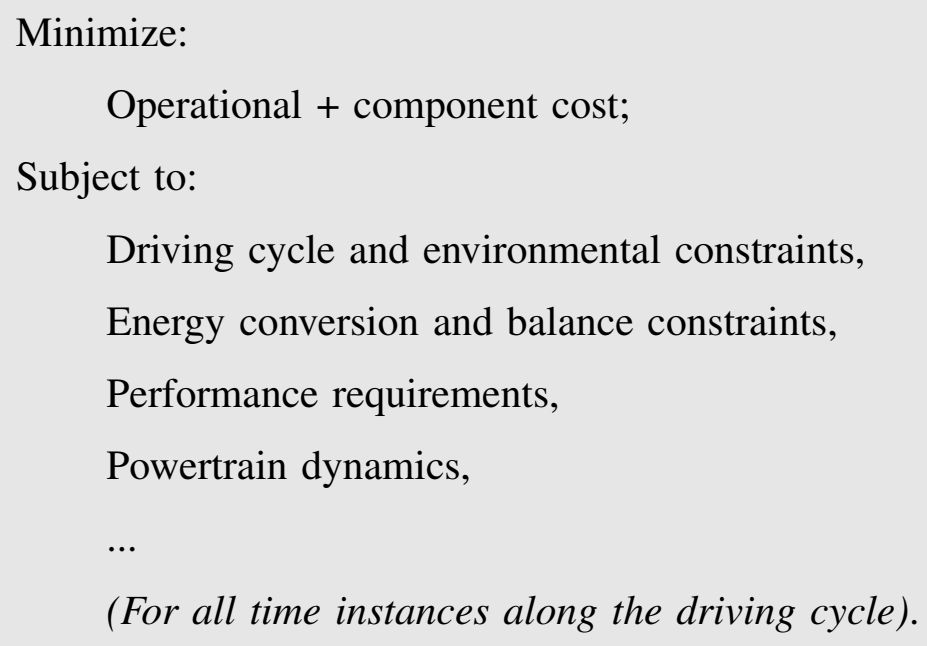

component costs of the vehicle. Thus the optimal buffer size - in terms of power rating and energy capacity - is the one that provides the optimal tradeoff between component cost and operational cost within the lifetime of the vehicle. Without now going into mathematical details, the optimal buffer sizing problem can be formulated as an optimization problem (see Table I) with two weighted objectives and several constraints.

The discussion of this design example is resumed towards the end of the article, when the needed tools have been provided. It is used as one of the case studies to illustrate how the optimization problem is formulated and solved, thus providing insights about the properties of the stated design problem.

\section{Modeling of energy and cost}

To study energy efficiency aspects of powertrains, it is necessary to have a clear picture of the power flows in the driveline. It turns out that a simple and basic representation of the driveline components, based on these power flows, is sufficient for the intended studies. The starting point 
is to represent the powertrains, as depicted in Figures S1 and S2, in a block diagram form, as seen in Figure 2. Here, the two primary power sources, namely fuel $P_{f}$ and electric power $P_{e}$ (in the case of a plug-in hybrid), are shown at the left. Then it is seen how power is transformed and combined to finally propel the vehicle. Note that the arrows indicate positive power flow, where power may sometimes flow in the opposite direction between components, for example during braking. Based on this description of the powertrains, the design procedure can be formulated as three steps.

1) Define models for each of the standard component types depicted in Figure 2. Models describing the components' power flows, cost and weight as functions of size are needed.

2) Define an overall powertrain model based on the component models, as well as information about the powertrain configuration (the interconnections of components).

3) Based on the powertrain model, state an optimization problem that includes both energy management and sizing.

Each of these steps is described in detail below, and the necessary model approximations are clarified.

\section{Component models}

Because the optimization is stated in terms of power flows and stored energy, the generic component model $X$ is stated in terms of input/output powers $\left(P_{X 1}\right.$ and $\left.P_{X 2}\right)$, power dissipation $\left(P_{X d}\right)$, and stored energy $\left(E_{X}\right)$ with corresponding power flow $\left(P_{X s}\right)$. For notational convenience, the time argument $t$ of the power and energy variables are omitted). The model is shown graphically in Figure 3, together with the fundamental energy and power balances. Once again, it is noted that the direction of the arrows indicate positive power flow. Within this general model class, specific details are provided for each component. First, the dissipative power is approximated as a convex function of the other component variables. Second, in order to allow the study of sizing problems, a linear scaling with respect to component size is performed on power flows and stored energy. Finally, convex inequality constraints are used to express component

limitations during operation. Below follows a description of how component models can be 
defined according to these principles, along with motivations for these modeling assumptions.

\section{Cost and weight modeling}

The scaling of components affects not only power and energy. It is also assumed that the size of the component affects both weight and cost in a linear fashion similarly as in, for example, [23]; for rotating machines, it is shown later how also moment of inertia is scaled. Hence, for component $X$, the weight $m_{X}$ and the cost $c_{X}$ depend on the size scaling factor $s_{X}$

$$
\begin{gathered}
m_{X}=m_{X}\left(s_{X}\right), \\
c_{X}=c_{X}\left(s_{X}\right),
\end{gathered}
$$

where $m_{X}$ and $c_{X}$ are affine in $s_{X}$. It is clear that the assumption of linearity in both weight and cost as a function of size is quite strong. If more accurate models are available at an early design stage, it is straightforward to relinearize these models and repeat the optimization step to obtain more accurate estimates of the optimal solution. Details on cost modeling are given in the case studies involving, for example, calculation of depreciation over a specified lifetime.

\section{Machine model}

The models for the combustion engine, electrical motor/generator, transmission, and the combined engine/generator unit (used in series type powertrains), are similar. The model for the electrical machine (M) is shown first. The generic model depicted in Figure 3 specializes to the following model

$$
\begin{aligned}
P_{M e} & =P_{M m}+P_{M d}-P_{M s}, \\
\dot{E}_{M} & =-P_{M s}
\end{aligned}
$$

where the subscripts $e$ and $m$ refer to electrical and mechanical power respectively.

In order to address the sizing problem, it is assumed that the power flows and the energy scale linearly with component size. Hence, the dissipation power $P_{M d}$ and the stored energy $E_{M}$ are defined in two steps: first for a nominal machine model, then for a scaled version. The 
nominal model is defined by the generic model equations and the following expressions for $P_{M d}$ and $E_{M}$

$$
P_{M d}=f_{M}^{\omega_{M}}\left(P_{M m}\right)
$$

where $f_{M}^{\omega_{M}}$ is nonnegative and convex, and

$$
E_{M}=\frac{1}{2} J_{M}^{0} \omega_{M}^{2}
$$

The rotational energy is given in terms of the moment of inertia $J_{M}^{0}$ and the machine speed $\omega_{M}$. The essential step here is to find an approximation that permits the dissipative power to be described by the convex function $f_{M}^{\omega_{M}}$, often parameterized in angular speed $\omega_{M}$, which is indicated by the superscript. The description of input power (or torque) as an affine, speeddependent function of output power (torque) (referred to as Willans lines), is a special case of the model considered here. More accurate models describing the losses as quadratic functions are also covered by the convex loss model. There are ample examples of this type of models for both electric machines and combustion engines; see [4] and references therein.

The scaled model is derived from the nominal model by allowing both power and energy to scale linearly with respect to the component size $s_{M}$, which is equal to 1 for the nominal model

$$
\begin{aligned}
P_{M d} & =s_{M} \cdot f_{M}^{\omega_{M}}\left(\frac{P_{M m}}{s_{M}}\right), \\
E_{M} & =\frac{1}{2} J_{M}\left(s_{M}\right) \omega_{M}^{2},
\end{aligned}
$$

with $J_{M}$ affine in $s_{M}$. The new dissipation function $s_{M} f_{M}^{\omega_{M}}\left(\frac{P_{M m}}{s_{M}}\right)$ is convex since it is the perspective function of the convex function $f_{M}^{\omega_{M}}$ [24]. The interpretation of (7) is that the dissipation characteristics are valid for the normalized power quantities (divide both sides of (7) by $s_{M}$ to see this). This means that nominal, nonlinear efficiency maps in the speed-torque plane, often used to describe electrical machines and combustion engines, are applied to scaled components by stretching or compressing the maps in the torque dimension (see Case study 2 for an illustration of this).

Linear scaling can provide good estimates if the sizes are not too far from the nominal values; this has already been shown in previous work on component sizing, see for example [13], 
[25], [26], [27], [11]. However, if the optimization gives a size which is far from the nominal value, a new nominal model can be generated and the optimization can be repeated, as in [5].

In addition to the model equations given above, the models are subject to constraints during operation. For the machine model, the following (speed dependent) constraints concern the allowed power levels

$$
P_{M, \min }^{\omega_{M}}\left(s_{M}\right) \leq P_{M m} \leq P_{M, \max }^{\omega_{M}}\left(s_{M}\right),
$$

where $P_{M, \text { min }}^{\omega_{M}}$ and $P_{M, \text { max }}^{\omega_{M}}$ are affine in $s_{M}$. In summary, the electrical machine model is given by equations (3), (4), (7)-(9), in combination with the mass and cost models (1), (2) with $X=M$.

The modeling carried out above for the electrical motor can be repeated with minor changes for the other rotational components. The important step is to find a convex, approximate loss model for each component as discussed further in the case studies. To avoid too much repetition at this stage, the models thus obtained are summarized in Figure 4. Note that the stored rotational energies in the models are not "pure" states, as they represent mechanically coupled inertias that are related to the vehicle dynamics. The power flow $P_{s}$ is, in fact, determined by "differential causality" from the pre-determined velocity trajectory. Since the dominating inertia is the vehicle itself, it is common to neglect the machine inertias. However, the storage term has been kept in the models to show the connection with the buffer model to come next, and to allow treatment of other problem setups, as discussed at the end of the article.

Finally, note that as a consequence of the required convexity of the dissipation functions, the machine models are assumed to describe the components in operation, implying that the models include idle losses of the machines. To allow switching off the machines, giving zero losses, a separate mechanism is needed, as discussed later. 


\section{Energy buffer model}

The energy buffer model, covering both battery and supercapacitor storage, is based on the same generic model as the rotational machines. The basic equations are thus given by

$$
\begin{aligned}
P_{B c} & =P_{B t}+P_{B d}-P_{B s}, \\
\dot{E}_{B} & =-P_{B s},
\end{aligned}
$$

where subscripts $c$ for charging, $t$ for terminal have been used, and the standard sign convention that the battery/capacitor power $P_{B s}$ is positive when discharging has been adopted. Looking at the modeling details, the fundamental assumption is that the buffer is built from cells that are described by an open circuit voltage (OCV) in series with an internal resistance $R$. The OCV $u$ is assumed to vary linearly with the charge $q$, so that the cell is described by the equations

$$
\begin{aligned}
& u=u_{0}+\frac{1}{C} q, \\
& \dot{q}=-i,
\end{aligned}
$$

where $i$ is the cell current. With this cell model, common special cases can be treated in a unified manner: with $u_{0}=0$, the model describes a supercapacitor; with $C=\infty$, a battery with constant OCV can be modeled.

The nominal model is obtained by aggregating $n_{0}$ cells (the particular arrangement in series/parallel is irrelevant from a power perspective). The model is described by the following equations for the stored energy and the dissipation power

$$
\begin{aligned}
E_{B} & =n_{0} \cdot \int_{0}^{q} u\left(q^{\prime}\right) d q^{\prime}=n_{0}\left(u_{0} q+\frac{1}{2 C} q^{2}\right)=n_{0} \frac{1}{2} C\left(u^{2}-u_{0}^{2}\right), \\
\dot{E}_{B} & =\frac{d E_{B}}{d q} \cdot \dot{q}=-n_{0} \cdot u \cdot i=-P_{B s}, \\
P_{B d} & =n_{0} R i^{2}=R \frac{P_{B s}^{2}}{n_{0} u^{2}}=R \frac{P_{B s}^{2}}{n_{0} u_{0}^{2}+\frac{2}{C} E_{B}} \triangleq f_{B}\left(P_{B s}, E_{B}\right) .
\end{aligned}
$$

The function $f_{B}$ is quadratic-over-linear and therefore has the required convexity property (see [24]).

The scaled model is needed to model a battery/capacitor that is larger or smaller than the nominal one. Therefore, define a (real) scaling factor $s_{B}$ and replace $n_{0}$ with $s_{B} \cdot n_{0}$ in the 
model

$$
\begin{aligned}
E_{B} & =s_{B} \cdot n_{0} \cdot \int_{0}^{q} u\left(q^{\prime}\right) d q^{\prime}=s_{B} \cdot n_{0} \frac{1}{2} C\left(u^{2}-u_{0}^{2}\right), \\
P_{B d} & =R \frac{P_{B s}^{2}}{s_{B} n_{0} u_{0}^{2}+\frac{2}{C} E_{B}}=s_{B} R \frac{\left(P_{B s} / s_{B}\right)^{2}}{n_{0} u_{0}^{2}+\frac{2}{C}\left(E_{B} / s_{B}\right)}=s_{B} \cdot f_{B}\left(\frac{P_{B s}}{s_{B}}, \frac{E_{B}}{s_{B}}\right) .
\end{aligned}
$$

The function $s_{B} f_{B}\left(\frac{P_{B s}}{s_{B}}, \frac{E_{B}}{s_{B}}\right)$ is convex, seen either directly from the quadratic-over-linear expression, or from the fact that it is the perspective function of the convex function $f_{B}(\cdot, \cdot)$.

Finally, the energy buffer has constraints on both power losses and energy (or charge); both scale linearly with size. In addition, there are constraints on charging

$$
\begin{gathered}
P_{B d} \leq s_{B} \cdot n_{0} R i_{\text {max }}^{2} \\
s_{B} \cdot n_{0} e_{\min } \leq E_{B} \leq s_{B} \cdot n_{0} e_{\max } \\
P_{B, \min } \leq P_{B c} \leq P_{B, \max }
\end{gathered}
$$

Here, $\left[e_{\min }, e_{\max }\right]$ is the allowed range of stored energy per cell, and $i_{\max }$ is the maximum allowed current magnitude. If the maximum magnitude of charging and discharging currents are different, then the constraint (19) can be replaced by the inequalities

$$
n_{0} i_{\min } \sqrt{s_{B}\left(\frac{2 E_{B}}{n_{0} C}+s_{B} u_{0}^{2}\right)} \leq P_{B s} \leq n_{0} i_{\max } \sqrt{s_{B}\left(\frac{2 E_{B}}{n_{0} C}+s_{B} u_{0}^{2}\right)} .
$$

Here, the lower limit is convex and the upper limit is concave due to the fact that the geometric mean is a concave function of its arguments (and $i_{m i n}<0$ ). The lower bound on $P_{B c}$ in (21) would normally be zero but could be negative in studies where power delivery to the grid is allowed. The model is depicted in Figure 5.

\section{Vehicle model}

So far, the models described all motivate the term "component models". The model of the vehicle's power use, to be described next, is not really a component model, since the scaling parameters of the powertrain components affect the overall vehicle model. Despite this, it is convenient to describe the vehicle model in an analogous way to the components, thus adhering to the general framework. Hence, the vehicle model is a special case of the generic model in 
Figure 3

$$
\begin{aligned}
& P_{V}=P_{V d}-P_{V s}, \\
& \dot{E}_{V}=-P_{V s},
\end{aligned}
$$

where the subscript $V$ stands for vehicle.

The nominal model is obtained by specifying the dissipative terms due to air resistance, rolling resistance, and braking; the energy is comprised of kinetic and potential energy

$$
\begin{aligned}
P_{V d} & =\left(F_{v}^{a}+m_{V}^{0} a_{r}\right) \cdot v+P_{V b}, \\
E_{V} & =\frac{1}{2} m_{V}^{0} v^{2}+m_{V}^{0} g h .
\end{aligned}
$$

Here $F_{v}^{a}$ is the aerodynamic drag, $a_{r}=c_{r} \cos \alpha$ is the retardation due to rolling resistance $(\alpha$ is the road inclination), and $P_{V b}$ is the braking power. The second equation contains the kinetic energy expressed in mass $m_{V}^{0}$ and speed $v$, and potential energy depending on altitude $h$.

The scaled model for the vehicle depends on the scaled components with their weight models

$$
\begin{aligned}
P_{V d} & =\left(F_{v}^{a}+m_{V}(s) a_{r}\right) \cdot v+P_{V b}, \\
E_{V} & =\frac{1}{2} m_{V}(s) v^{2}+m_{V}(s) g h
\end{aligned}
$$

where $m_{V}(s)$ is affine in $s=\left(s_{E}, s_{G}, s_{B}, s_{M}, s_{T}\right)$. It would also be possible to include an additional scaling factor for a part of the vehicle that does not depend directly on powertrain components, such as the mass of the chassis. This idea is not pursued further here, however.

Finally, the only constraint applicable for the vehicle model is the sign of the braking power

$$
P_{V b} \geq 0
$$

\section{Powertrain modeling}

The modeling of a vehicle powertrain is done by combining component models in a power flow diagram. The overall model is then defined by all component models (equations 
+ constraints), plus the interconnections. The driving mission is defined by a velocity profile, which combined with a gear switching sequence define the angular speeds of all mechanically connected machines. This constraint is depicted as in the series powertrain example shown in Figure 6: the components that are mechanically connected are marked by the dashed lines as "coupled". In this figure, the gear switching sequence $r(\cdot)$ is indicated as an external signal; the meaning is that the gear switching is assumed to be predetermined from the driving profile and not included in the optimization problem.

Figure 6 also includes two binary on/off signals $\sigma_{C}$ and $\sigma_{G}$. These external signals control whether charging is allowed (the charger block marked with $\mathrm{C}$ is trivial, containing only the on/off switch and possibly a constant efficiency), and whether the engine/generator unit is operating or not. If $\sigma_{X}=1$, the component equations are included in the overall model. If, on the other hand, $\sigma_{X}=0$, the component equations are excluded and the connecting power flows are set to 0 . The on/off signals provide the mechanism referred to previously, which takes care of the cases when there is a need to switch off a component, for example the ICE. The convex models are formulated only for the case when the machine(s) are in operation. The disadvantage is that the switching decisions have to made beforehand, based on the driving cycle specification. This limitation is discussed further towards the end of the article.

The third step in the 3-step procedure outlined at the beginning of this section is to state the optimization problem in terms of the powertrain model compiled. This step is addressed in the next section.

\section{Optimization}

The powertrain model forms a set of constraints to be fulfilled during optimization. Continuing to use the example depicted in Figure 6 to illustrate the basic ideas, the objective to minimize has two components:

- The operational or energy cost is comprised of the costs for electric energy and fuel,

$$
c_{o p}=\int_{0}^{t_{f}}\left(\rho_{e} P_{e}(t)+\rho_{f} P_{f}(t)\right) d t
$$


where $\rho_{e}$ and $\rho_{f}$ are price parameters (currency/W) and the driving mission lasts for $t_{f} \mathrm{~s}$.

- The capital or component cost is the size dependent part of the cost for the powertrain components,

$$
c_{c a p}=\sum_{X} c_{X}\left(s_{X}\right)
$$

where the summation is over all driveline components that have been included in the model with sizing parameters.

By discretizing the variables in (30) with a discretization interval $\Delta t$, the integral is approximated as a sum, and the optimization problem can formally be stated as

$$
\begin{aligned}
& \text { minimize } \quad c=c_{o p}+c_{c a p}=\sum_{k=1}^{k_{f}}\left(\rho_{e} P_{e}(k)+\rho_{f} P_{f}(k)\right) \Delta t+\sum_{X} c_{X}\left(s_{X}\right) \\
& \text { subject to }\left\{\begin{array}{l}
\text { component equations and inequalities, } \\
\text { connection equations, } \\
\text { vehicle requirements, }
\end{array}\right.
\end{aligned}
$$

where the constraints could include, for example, performance requirements or conditions on sustained battery charge over the entire drive cycle. This point is illustrated in the case studies. The minimization is with respect to the variables in the problem, reflecting both the arbitration of the power flows and the component sizing. There is an important difference between the two types of variables: the component sizes $\left\{s_{X}\right\}$ express properties of the powertrain to hold over the entire driving mission, whereas the power and energy variables depend on time. Therefore, each such variable gives rise to a vector of optimization variables, with the number of entries $k_{f}=t_{f} / \Delta t$ depending on both discretization interval and the total time $t_{f}$ of the driving mission.

The statement of the optimization problem in (32) is too general to be useful, and therefore requires a more detailed discussion. In particular, it is shown in the next section how the problem can indeed be formulated as a convex optimization problem. See "Convex Optimization" for an introduction. 


\section{Convexification}

The optimization problem (32) makes reference to the powertrain model, including equations for components and their interconnections, as well as component constraints. For the series PHEV shown in Figure 6, adding the component models, as shown in Figure 7, gives a model suitable for optimization. For brevity, the gear switching signal has been omitted from the figure, and the external binary signals $\sigma_{C}$ and $\sigma_{G}$ have been included in the connecting power flows.

With reference to Figure 7, it will now be shown how the optimization problem can be formulated as a standard convex problem. The essential step is to provide a systematic way to handle the originally nonaffine (but convex) equality constraints, arising from the dissipation terms. The procedure consists of the following two steps

1) First it can be noticed that the power balance equation for the engine/generator unit,

$$
\sigma_{G} P_{f}=\sigma_{G}\left(P_{G d}+P_{G e}\right)=\sigma_{G}\left(s_{G} f_{G}\left(\frac{P_{G e}}{s_{G}}\right)+P_{G e}\right)
$$

is valid only when the EGU is on $\left(\sigma_{G}=1\right)$. Equation (33) can thus be used to replace $P_{f}$ in the objective function by the right hand side expression, giving

$$
c=\rho_{e} \sum_{\sigma_{C}(k)=1} P_{e}(k) \Delta t+\rho_{f} \sum_{\sigma_{G}(k)=1}\left(s_{G} f_{G}\left(\frac{P_{G e}(k)}{s_{G}}\right)+P_{G e}(k)\right) \Delta t+\sum_{X} c_{X}\left(s_{X}\right),
$$

where the power summations are carried out over time intervals when $\sigma_{C}=1$ and $\sigma_{G}=1$, respectively. From the assumptions, it follows that the objective is convex in the sizing variables and the (vector) variables $P_{e}, P_{G e}$. The same procedure would be applied to replace $P_{e}$, if a dissipation model for the charger had been included.

2) The second step amounts to compiling equality constraints from the power balances for all remaining components, and then to relax the dissipation equalities to inequalities. In 
the example, this results in

$$
\begin{gathered}
\sigma_{C} P_{e}=P_{B d}-P_{B s}+P_{B t} \geq s_{B} f_{B}\left(\frac{-\delta E_{B}}{s_{B}}, \frac{E_{B}}{s_{B}}\right)+\delta E_{B}+P_{B t}, \\
P_{B t}+\sigma_{G} P_{G e}=P_{M e}=P_{M d}-P_{M s}+P_{M m} \geq s_{M} f_{M}^{\omega_{M}}\left(\frac{P_{M m}}{s_{M}}\right)-P_{M s}+P_{M m}, \\
P_{M m}=P_{T d}-P_{T s}+P_{T 2} \geq s_{T} f_{T}^{\omega_{T}}\left(\frac{P_{T 2}}{s_{T}}\right)-P_{T s}+P_{T 2}, \\
P_{T 2}=P_{V d}-P_{V s} \geq\left(F_{v}^{a}+m_{V}(s) a_{r}\right) v-P_{V s} .
\end{gathered}
$$

Here, the notation $-P_{B s}=\delta E_{B}(k)=\left(E_{B}(k)-E_{B}(k-1)\right) / \Delta t$ has been used in (35), and the equalities for $P_{B d}, P_{M d}, P_{T d}$ and $P_{V b}$, respectively, have been relaxed (the latter implying that the braking power $P_{V b}$ has been removed from the problem). Due to the characteristics of the dissipation functions for the machine models in both objective and constraints and the "nested" structure of the inequality constraints, it can be shown that these relaxations do not change the properties of the optimal solution. This can be understood intuitively by the fact that energy would otherwise be wasted. See "Constraints Relaxation" for a more rigorous argument.

After having carried out the transformations in the two steps described above, the objective in (32) is expressed as a convex function of the optimization variables, and the constraints are given by the convex inequalities in (35)-(38). It now only remains to describe how to incorporate the specified driving cycle.

\section{The driving mission}

As has been mentioned already, the storage terms for the components that are mechanically connected to the wheels of the vehicle are not state variables in the usual sense. The reason is that it is assumed that the vehicle follows the driving profile exactly, and the way this is implemented is by "differential causality", meaning that the required tractive power is calculated by differentiating the speed (referred to as "backward simulation" [4]). In addition, and as indicated in the powertrain schematic, there are implicit constraints that couple mechanically connected machines.

In the example, there are three mechanically coupled storage variables, namely $P_{M s}, P_{T s}$, 
and $P_{V s}$. Each of them is defined by differentiating the respective energy variable

$$
\begin{aligned}
P_{M s} & =J_{M}\left(s_{M}\right) \omega_{M} \dot{\omega}_{M}, \\
P_{T s} & =J_{T}\left(s_{T}\right) \omega_{T} \dot{\omega}_{T}, \\
P_{V s} & =m_{V}(s) v \dot{v}+m_{V}(s) g \dot{h}=m_{V}(s) v \dot{v}+m_{V}(s) g v \sin \alpha .
\end{aligned}
$$

All these expressions are affine with respect to the sizing parameters, assuming the velocity profile $v$ and the gear sequence $r$ are known. By inserting discretized versions of them into the inequalities (35)-(38), the optimization problem is completely defined.

\section{Solving the optimization problem}

The final statement of the optimization problem will be formulated in terms of full vector variables, written in bold face and defined as, for example, $\boldsymbol{P}_{B t}=\left(P_{B t}(1), \cdots, P_{B t}\left(k_{f}\right)\right)$. The definitions of $\boldsymbol{P}_{e}$ and $\boldsymbol{P}_{G e}$ are sparse due to the on/off signals: $\boldsymbol{P}_{e}$ has entries $\sigma_{C}(k) P_{e}(k)$ and $\boldsymbol{P}_{G e}$ has entries $\sigma_{G}(k) P_{G e}(k)$. The optimization problem can now be stated as

$$
\begin{array}{ll}
\text { minimize } & c=\rho_{e} \Delta t \mathbf{1}^{T} \boldsymbol{P}_{e}+\rho_{f} \Delta t \mathbf{1}^{T}\left(\boldsymbol{\sigma}_{G} s_{G} f_{G}\left(\frac{\boldsymbol{P}_{G e}}{s_{G}}\right)+\boldsymbol{P}_{G e}\right)+\sum_{X} c_{X}\left(s_{X}\right) \\
\text { subject to } & \boldsymbol{P}_{e} \geq s_{B} f_{B}\left(\frac{-\delta \boldsymbol{E}_{B}}{s_{B}}, \frac{\boldsymbol{E}_{B}}{s_{B}}\right)+\delta \boldsymbol{E}_{B}+\boldsymbol{P}_{B t}, \\
& \boldsymbol{P}_{B t}+\boldsymbol{P}_{G e} \geq s_{M} f_{M}^{\boldsymbol{\omega}_{M}}\left(\frac{\boldsymbol{P}_{M m}}{s_{M}}\right)+J_{M}\left(s_{M}\right) \boldsymbol{\omega}_{M} \delta \boldsymbol{\omega}_{M}+\boldsymbol{P}_{M m}, \\
& \boldsymbol{P}_{M m} \geq s_{T} f_{T}^{\boldsymbol{\omega}_{T}}\left(\frac{\boldsymbol{P}_{T 2}}{s_{T}}\right)+J_{T}\left(s_{T}\right) \boldsymbol{\omega}_{T} \delta \boldsymbol{\omega}_{T}+\boldsymbol{P}_{T 2}, \\
& \boldsymbol{P}_{T 2} \geq\left(F_{v}^{a}+m_{V}(s) a_{r}\right) \boldsymbol{v}+m_{V}(s) \boldsymbol{v} \delta \boldsymbol{v}+m_{V}(s) g \boldsymbol{v} \sin \alpha, \\
& \text { component inequality constraints, } \\
& \text { vehicle requirements, }
\end{array}
$$

where 1 is a vector of 1's, $\sigma_{G}$ has entries $\sigma_{G}(k)$, and multiplication of the vector variables is interpreted component-wise. From this formulation it is seen that the problem is a standard convex optimization problem. The optimization variables are the scaling parameters $s=\left(s_{B}, s_{G}, s_{M}, s_{T}\right)$ and the power/energy vector variables $\boldsymbol{P}_{e}, \boldsymbol{P}_{B t}, \boldsymbol{P}_{G e}, \boldsymbol{P}_{M m}, \boldsymbol{P}_{T 2}, \boldsymbol{E}_{B}$. Notice once again that the 
binary signals $\sigma_{G}$ and $\sigma_{C}$ are assigned outside the optimization, thus determining both the variable $\boldsymbol{\sigma}_{G}$ and the sparseness patterns of $\boldsymbol{P}_{e}$ and $\boldsymbol{P}_{G e}$.

In order to solve this optimization problem, there are many open-source solvers to choose from. Some examples are SeDuMi [28] and SDPT3 [29]. There are also Matlab-based packages, like CVX [30], [31] and YALMIP [32], which can automatically transform the problem into, for example, a sparse matrix form before passing it to the solver. The case studies to be described next have been solved by using CVX, a package for specifying and solving convex programs. CVX offers modeling support in the form of disciplined convex programming, which means that the optimization problem entered is automatically checked to fulfill the conditions for a convex optimization problem in standard form.

\section{Applications}

The preceding sections showed how the combined sizing and energy management problem for hybrid electric powertrains can be formulated as convex optimization problems. This allows many different types of comprehensive feasibility and concept studies to be conducted with moderate computational demands. For example:

- Based on the fact that an optimal control strategy is computed as part of the solution, a "fair" comparison can be made between competing vehicle designs.

- The tradeoff between component and operational costs can be studied for different prices on fuel, electricity, batteries etc.

- The influence of performance requirements, other than energy efficiency, on optimal component sizing and total cost can be studied.

- Optimal design to achieve minimum "total cost of ownership" can be computed for different assumptions.

- The influence of driving patterns and charging possibilities on optimal powertrain configuration can be analyzed.

- The optimal tradeoff between onboard storage and charging facilities can be calculated. 
It is possible to extend the models and methods outlined above in several ways, as discussed in the final section. First, however, the method is illustrated by briefly presenting two case studies. The first case study describes the sizing of two energy buffers: a battery and a supercapacitor for a series hybrid powertrain on a city bus. The second case study is an investigation of the influence of driving patterns on the optimal design of a parallel passenger vehicle. More details on these and similar studies can be found in the references cited.

\section{Case study 1}

This case study concerns the optimal buffer sizing of an electrified city bus with a series powertrain configuration, and was introduced in the beginning of the article. More specifically, a double buffer system consisting of an energy-optimized battery and a supercapacitor is investigated. As mentioned previously, the bus can charge for $10 \mathrm{~s}$ at seven bus stops. In order to investigate the influence of charging infrastructure on the optimal buffer size, an additional charging scenario is considered, in which the time schedule allows the bus to charge for $10 \mathrm{~min}$ before starting the route. The magnitude of charging power is $200 \mathrm{~kW}$ for each charger. However, in the second scenario, the possibility of downsizing the chargers is also investigated, such that the magnitude of charging power is chosen in accordance with the optimal buffer size. It is assumed that the chargers have a constant and identical efficiency of $92 \%$.

\section{Powertrain setup and modeling}

The bus is equipped with a $150 \mathrm{~kW}$ diesel engine/generator unit (EGU) and a $200 \mathrm{~kW}$ electrical machine (EM), as illustrated in Figure 8. The efficiency of the power electronics is aggregated and reflected in the EM losses. Figure 9 depicts EM power loss as a function of mechanical power. The loss is a convex function of the power. A second order polynomial

$$
P_{M d}=b_{2}^{\omega_{M}} P_{M m}^{2}+b_{1}^{\omega_{M}} P_{M m}+b_{0}^{\omega_{M}},
$$

with speed-dependent coefficients $b_{j}^{\omega_{M}}$ is a good approximation for the losses [4]. The coefficients are obtained for several discrete (gridded) values of the EM speed and linearly interpolated between gridded values. The remaining EM modeling details are exactly as in (3, 4, 7-9), but 
with scaling parameter $s_{M}=1$. The EM is connected to the wheels through a differential gear, without using a gearbox. The efficiency of the differential gear is assumed constant over different speeds and torques.

The EGU's power losses are modeled as quadratic in generator power

$$
P_{G d}=a_{2} P_{G e}^{2}+a_{1} P_{G e}+a_{0} .
$$

Further details on the validity of the model can be found in [21]. Constraints are invoked on the generator power, as depicted in Figure 4.

The battery pack is comprised of high energy lithium-ion cells, Saft VL 45E [33]. The cell's open circuit voltage (see Figure 10) is approximated as affine in state-of-charge (SOC), which gives a reasonable fit within the operating SOC range. In order to prolong battery life, the SOC range is limited between 20\%-80\%. The supercapacitor cell is a Maxwell BCAP2000 P270 [34] and the basic equations describing the cells are (10)-(21). A battery price of $500 \mathrm{EUR} / \mathrm{kWh}$ and a supercapacitor price of $10000 \mathrm{EUR} / \mathrm{kWh}$ are assumed. The remaining vehicle details, diesel fuel and electricity prices, and depreciation expenses are exactly as in [35].

\section{Engine on/off control}

To improve HEV efficiency it is recommended to turn the ICE off at low speeds and power demands where the ICE is least efficient [4]. Based on this concept, a heuristic strategy, suggested in [21], is applied. Being heuristic, this strategy does not guarantee global optimality. However, it has been observed [21], [36] that for series PHEV powertrains this heuristic gives results that are close to optimal. The strategy considers turning the engine on when the demanded power of a baseline vehicle, where components are not scaled, exceeds a threshold $P_{o n}$. The convex problem (now including the scaling variables) is solved for several gridded power thresholds to find the optimal threshold $P_{o n}^{*}$. Expressed in mathematical terms the on/off control is defined as

$$
\sigma_{G}= \begin{cases}1, & P_{V}\left(s_{B 1}=1, s_{B 2}=1\right)>P_{o n}^{*} \\ 0, & \text { otherwise }\end{cases}
$$

where $s_{B 1}$ and $s_{B 2}$ are supercapacitor and battery scales. 
TABLE II

OPTIMAL RESULTS FOR TWO DIFFERENT CHARGING SCENARIOS.

\begin{tabular}{l|lll}
\hline Charging scenario & 7 chargers & 1 charger & \\
\hline Supercapacitor energy & 0.8 & 0.4 & $\mathrm{kWh}$ \\
Available battery energy & 2.4 & 15.6 & $\mathrm{kWh}$ \\
Total cost & 31.7 & 16.3 & $\mathrm{EUR} / 100 \mathrm{~km}$ \\
Diesel fuel consumption & 16.4 & 0 & $1 / 100 \mathrm{~km}$ \\
Charging power & 200 & 121 & $\mathrm{~kW}$ \\
\hline
\end{tabular}

\section{Convex optimization problem}

For a given engine on/off and charging sequence, the convex optimization problem is very similar to (40)-(44) with only small differences which arise because of the double buffer system. The buffer power, in this case, is the cumulative power from the two packs, and thus the variable $\boldsymbol{P}_{B t}$ becomes $\boldsymbol{P}_{B t 1}+\boldsymbol{P}_{B t 2}$. Similarly, the grid power $P_{e}$ is the sum of charging powers of the two packs. This is reflected in the objective function by changing the operational cost for electricity to

$$
\frac{\rho_{e}}{\eta_{e}} \Delta t\left(\mathbf{1}^{T} \boldsymbol{P}_{e 1}+\mathbf{1}^{T} \boldsymbol{P}_{e 2}\right)
$$

where the charger losses have been included by dividing by the efficiency $\eta_{e}$. There will be one inequality constraint (41) for each of the packs and, similarly, there will be two sets of buffer constraints (19)-(22).

\section{Optimal results}

The optimal results for the PHEV design for the two charging scenarios are in Table II. The optimal buffer size depends strongly on the charging infrastructure. When the charging durations are short, as in the scenario with seven chargers, it is optimal to include a larger supercapacitor pack of about $0.8 \mathrm{kWh}$ and a relatively small battery of about $2.4 \mathrm{kWh}$. The bus then consumes $16.4 \mathrm{l} / 100 \mathrm{~km}$ diesel fuel, with a total cost of $31.7 \mathrm{EUR} / 100 \mathrm{~km}$. In the second 
scenario, where charging duration is longer, it is optimal to include a supercapacitor pack of half the size (about $0.4 \mathrm{kWh}$ ) and a much larger battery (about $15.6 \mathrm{kWh}$ ). In this case, the bus can drive entirely as electric vehicle at about half the cost $(16.3 \mathrm{EUR} / 100 \mathrm{~km})$. For this infrastructure, a charger that can provide $120 \mathrm{~kW}$ is sufficient.

The optimal buffer SOC trajectories and operating points are detailed in Figure 11. The supercapacitor is used at high power transients, where it is more efficient than the battery. This behavior is particularly evident in the first charging scenario, where the grid charging energy is almost entirely accumulated by the supercapacitor. Although used at high power, the supercapacitor is still operated far from its power limits. Therefore, it can be concluded that the supercapacitor is mainly sized by the driving cycle's energy requirements. On the contrary, the battery in the first charging scenario is sized by the power because the battery does not use more than $50 \%$ of its available SOC range. It also indicates that an energy-optimized battery is not a suitable choice for short, high-power charging intervals. The energy-optimized battery is a better choice in the second charging scenario, where its entire available SOC range is used. The supercapacitor, in this case, assists the battery when high power is demanded, beyond what can be delivered by the battery alone.

For each charging scenario, the convex problem was solved 30 times to obtain the optimal power threshold, which decides the engine on/off control. The computation time of the convex problem is 1-2 minutes on a standard PC (2.67 Ghz Dual Core CPU and 4 GB RAM).

\section{Case study 2}

The second case study concerns optimal component sizing of a plug-in hybrid electric passenger car with a parallel architecture. The components to be sized are the ICE, EM, and battery, taking into account performance requirements. The optimization gives the optimal component sizes and energy management for a certain driving cycle. To find component sizes, which are optimal with respect to the entire vehicle lifetime, would require knowing the lifetime driving pattern of the vehicle. However, since this is not possible, a long driving cycle that reflects real-life driving patterns is used. Here, data from $712 \mathrm{~km}$ of real driving during a month 
in Gothenburg is used. It is assumed that the driver can charge the battery during any parking time that lasts longer than 10 minutes. During the available parking periods, it is assumed that the vehicle is charged with constant current and power. It is also assumed that the chargers have a constant efficiency of $95 \%$.

\section{Powertrain setup and modeling}

The baseline vehicle has a $65 \mathrm{~kW}$ ICE and a $35 \mathrm{~kW}$ EM. The EM is connected to the wheels through a differential gear with a constant efficiency. The EM losses, including losses from the power electronics, are approximated well with a second order polynomial, similar to (45). The remaining EM modeling details are as described earlier, including the scaling parameter $s_{M} \cdot$

The fuel power $P_{f}$ of the ICE depends on engine torque and speed, and is investigated by engine experiments at steady state. The fuel power at each engine speed is approximated with a second-order polynomial in mechanical power as

$$
P_{f}=c_{2}^{\omega_{E}} P_{E m}^{2}+c_{1}^{\omega_{E}} P_{E m}+c_{0}^{\omega_{E}}
$$

where the coefficients $c_{j}^{\omega_{E}}$ are functions of speed, and are calculated in a similar way as $b_{j}^{\omega_{M}}$ for the EM. It is assumed that losses are linearly related to the scaling parameter, resulting in a model that is similar to the standard machine model. The speed-dependent constraints on power for the EM and ICE model are shown in Figure 4.

A heuristic, similar to one used in case study 1, is used to switch the ICE on/off. Each time the power of the baseline vehicle (where the components are not scaled) exceeds a threshold $P_{\text {on }}$, the engine is turned on. The convex problem is solved for several values of the power thresholds $P_{\text {on }}$ to find the optimal value.

The battery pack used in this study is similar to the one used in case study 1, with the SOC range restricted to be between $20 \%$ and $80 \%$. 
A long driving cycle can reflect real-life driving, but may not include situations that require high performance. Since performance is considered an important vehicle attribute by many drivers, it is desirable to include it in the component sizing problem. All electric range (AER), charge sustaining top speed, and acceleration at different speeds are examples of performance requirements that would be considered. Some examples of acceleration and top speed requirements are illustrated in Figure 12 a. Different performance requirements are obtained by scaling the baseline curve by $\alpha_{p}$ and changing the top speed. The requirements on acceleration at different speeds can be fulfilled by different combinations of ICE and EM sizes. Therefore, to include this constraint in the problem, the acceleration requirement is converted into a performance cycle, as illustrated in Figure $12 \mathrm{~b}$, and is appended to the real-life driving cycle. The performance cycle is simply the speed profile (from zero to top speed) obtained by following the maximum acceleration curve for the considered $\alpha_{p}$ value, assuming zero road gradient. An alternative interpretation/representation of the performance cycle is also possible by, for example, translating the requirement to ascent capability.

\section{Gear selection}

The engine is connected to the wheels through a 6 speed gearbox with a constant efficiency of $97 \%$. In general, gear shifting strategies that are functions of unknown optimization variables, such as torque, may lead to a non convex problem. The alternative is to select the gears outside the convex optimization from a speed-dependent hysteresis model applied to the known driving profile. Since this strategy does not consider the engine peak power, it can highly influence the optimal size of the ICE. Therefore, the strategy is modified so that, for high acceleration demands, the gear shifting happens at higher speeds. The details of this strategy are provided in [37]. 


\section{Optimal results}

The optimal PHEV designs are presented here for different performance requirements, with more details to be found in [37]. To see the effect of AER requirements on the component sizing and cost, the optimization is solved with different fixed battery sizes. The acceleration requirement is kept at the baseline $\alpha_{p}=1$, and the top speed requirement is $120 \mathrm{~km} / \mathrm{h}(\approx 33 \mathrm{~m} / \mathrm{s})$. The results are presented in Figure 13, and show that the increased battery capacity decreases the operational cost but increases the component costs linearly. The optimal battery size is 5.2 $\mathrm{kWh}$, which is equivalent to approximately $20 \mathrm{~km}$ of AER. For larger battery sizes, the increase in cost of components is higher than the gain in operational cost. However, the curve between 15 to $35 \mathrm{~km}$ AER is rather flat, indicating that in this range of AER the cost is not very sensitive to the requirement on AER. As shown in Figure 14, a $30 \%$ reduction in battery price or $30 \%$ more expensive fuel price increases the optimal AER about $50 \%$.

The sizing problem can also be viewed as a multi objective optimization problem, including the component cost and the operational cost. The weighting factor $w_{\text {comp }}$, used to include the two objectives in the problem, is calculated for a fixed lifetime and yearly driving length. However, in real-life, this value is different for different vehicles. By varying the weighting factor, a Pareto front is obtained for the optimal operational cost vs. the optimal component costs. This is shown in Figure 15 for different acceleration requirements and a maximum speed of $120 \mathrm{~km} / \mathrm{h}$. As expected, component and operational costs can be seen to be conflicting objectives. In other words, components that contribute to increased component costs, mainly the battery and EM, can lower the operational cost. For a vehicle with a longer lifetime driving distance, the value of the weighting factor is smaller, and therefore, it is optimal to pay more for the components and get a lower operational cost, compared to a vehicle with a shorter lifetime driving distance. Naturally, higher performance requirements increase both the component and operational costs. 


\section{Discussion and conclusions}

This article presented a framework to study design tradeoffs in the search for electromobility solutions based on approximate modeling of the power flows in the powertrain as a function of component sizes. An important consequence of the modeling assumptions is that the optimal energy management and component sizes can be computed simultaneously in a convex program. This means that competing designs can be evaluated in an objective way, avoiding the influence of a separate control system design. The fact that the optimization problem is convex allows large problems to be solved with moderate computational resources, and this can be exploited by, for example, running optimizations over very long driving cycles. The problem formulation also admits design decisions for the charging infrastructure to be included in the optimization.

There are, of course, limitations with the presented approach. Perhaps the most important is that integer variables cannot be treated within the convex problem. For example, the decision to turn on or off the combustion engine has to be decided outside the optimization. One way to do this is to use a simple rule, parameterized in terms of a power threshold, and then search for optimal parameters in an "outer optimization loop". For some problems, this approach leads to nearly optimal solutions [36]. Similarly, the gear switching has to be predetermined, based on a characterization of the driving cycle and the baseline powertrain. One way to avoid this step is to perform the optimization study with a continuously variable transmission (CVT), where the choice of "gear" can be treated as part of the convex optimization problem.

A second potential limitation is the range over which the linear component scaling is sufficiently accurate for optimal design decisions. However, if necessary, the scaling model can be relinearized about a different component size and the optimization, which is convex, can be solved repeatedly, until the simplified scaling model is in fact close to the optimum. This iteration, described in the modeling section, is a feasible approach because the primary problem is convex. A third potential limitation of the method is the accuracy of the convex loss models, but since dissipation functions often are not required to be highly accurate to function appropriately (for example, the Willans approximation [4]), this is not a great concern. 
The presented approach can be extended in various directions to treat more realistic, and therefore more complex, problems. A few of these possibilities will be briefly described.

Continuously variable transmissions $(C V T)$ : As has been mentioned already, it is possible to extend the method to also treat CVTs. The benefit of doing so is that the decision of gear switching is replaced by the choice of the continuous speed ratio, which can be included in the convex optimization. When including a CVT, the driveline is decoupled into two parts, and the rotational energy of the part including the engine is now treated as a pure state variable. As a consequence, the engine losses and constraints have to be expressed as convex functions of the rotational energy (or square of angular speed). This approach is worked out in detail in [38].

Thermal states: Limiting battery or supercapacitor temperature is an essential task of the control system. It is possible to include a thermal state with simple dynamics driven by the power dissipation $P_{B d}$ in the convex model. In this way, the optimal energy management can be required to keep the buffer temperature below a certain maximum temperature. An example study can be found in [35].

Battery wear: When sizing a battery for an HEV application, a relevant question is whether to choose one large battery sized to survive the entire lifespan of the vehicle, or several smaller replaceable batteries which could be operated at higher c-rates. Such studies necessitate a battery wear model that describes battery aging as a function of several variables. A complete wear model is still an ongoing research area, but variables that are typically considered important include c-rate, temperature, depth of discharge and state of charge. A simple wear model based on weighted c-rate has been studied in [39], while in [40] it has been shown that this model can also be used in convex optimization.

There are other possible directions for further work based on the presented approach. So far, fairly simple problems related to the charging infrastructure have been studied. Other scenarios include, for example, more detailed charger models or the possibility to transfer stored electrical energy in the vehicle back to the grid. Another area for further investigations is the use of other types of driving cycles. An alternative to the exactly specified velocity profile is to define a nominal velocity profile that allows some deviation in order to include an "intelligent 
driver" in the problem formulation. 


\section{References}

[1] C.C. Chan. Special issue on electric, hybrid, and fuel cell vehicles. Proceedings of the IEEE, 95(4), 2007.

[2] A.G. Boulanger, A.C. Chu, S. Maxx, and D.L. Waltz. Vehicle Electrification: Status and Issues. Proceedings of the IEEE, 99(6):1116-1138, June 2011.

[3] A. Sciarretta and L. Guzzella. Control of hybrid electric vehicles. IEEE Control Systems Magazine, pages 60-70, November 2007.

[4] L. Guzzella and A. Sciaretta. Vehicle Propulsion Systems. Springer Verlag, Berlin Heidelberg, 3rd edition, 2013.

[5] D. Assanis, G. Delagrammatikas, R. Fellini, Z. Filipi, J. Liedtke, N. Michelena, P. Papalambros, D. Reyes, D. Rosenbaum, A. Sales, and M. Sasena. An optimization approach to hybrid electric propulsion system design. Journal of Mechanics of Structures and Machines, Automotive Research Center Special Edition, 27(4):393-421, 1999.

[6] V. Galdi, L. Ippolito, A. Piccolo, and A. Vaccaro. A genetic-based methodology for hybrid electric vehicles sizing. Soft Computing, 5(6):451-457, 2001.

[7] L. Wu, Y. Wang, X. Yuan, and Z. Chen. Multiobjective optimization of HEV fuel economy and emissions using the self-adaptive differential evolution algorithm. IEEE Transactions on Vehicular Technology, 60(6):2458-2470, 2011.

[8] H. Fathy, P.Y. Papalambros, and A.G. Ulsoy. On combined plant and control optimization. In 8th Cairo University International Conference on Mechanical Design and Production, Cairo University, 2004.

[9] D.L. Peters, P.Y. Papalambros, and A.G. Ulsoy. Sequential co-design of an artifact and its controller via control proxy functions. Mechatronics, 23(4):409-418, 2013.

[10] J.A. Reyer and P.Y. Papalambros. Combined optimal design and control with application to an electric DC motor. Journal of Mechanical Design, 142(2):183-191, 2002.

[11] Z. Filipi, L. Louca, B. Daran, C.-C. Lin, U. Yildir, B. Wu, M. Kokkolaras, D. Assanis, H. Peng, P. Papalambros, J. Stein, D. Szkubiel, and R. Chapp. Combined optimisation of design and power management of the hydraulic hybrid propulsion system for the $6 \times 6$ medium truck. International Journal of Heavy Vehicle Systems, 11(3/4):372, 2004. 
[12] M.J. Kim and H. Peng. Combined control/plant optimization of fuel cell hybrid vehicles. In Proceedings of the 2006 American Control Conference Minneapolis, Minnesota, USA, June 14-16 2006.

[13] O. Sundström, L. Guzzella, and P. Soltic. Torque-assist hybrid electric powertrain sizing: From optimal control towards a sizing law. IEEE Transactions on Control Systems Technology, 18(4):837-849, July 2010.

[14] E.D. Tate and S. Boyd. Finding ultimate limits of performance for hybrid electric vehicles. SAE Transactions, 1998.

[15] M. Back, M. Simons, F. Kirschbaum, and V. Krebs. Predictive control of drivetrains. In Proc. IFAC World Congress, Barcelona, Spain, 2002.

[16] S. Terwen, M. Back, and V. Krebs. Predictive powertrain control for heavy duty trucks. In Proc. IFAC Symposium on Advances in Automotive Control, Salerno, Italy, 2004.

[17] M. Koot, J.T.B.A. Kessels, B. DeJager, W.P.M.H. Heemels, P.P.J. VandenBosch, and M. Steinbuch. Energy management strategies for vehicular electric power systems. IEEE Transactions on Vehicular Technology, 54(3):771-782, May 2005.

[18] R. Beck, A. Bollig, and D. Abel. Comparison of two real-time predictive strategies for the optimal energy management of a hybrid electric vehicle. Oil \& gas science and technology - Rev. IFP, 62(4):635-643, 2007.

[19] J.T.B.A. Kessels, D.L. Foster, and P.P.J. van den Bosch. Integrated powertrain control for hybrid electric vehicles with electric variable transmission. 2009 IEEE Vehicle Power and Propulsion Conference, pages 376-381, September 2009.

[20] N. Murgovski, L. Johannesson, J. Hellgren, B. Egardt, and J. Sjöberg. Convex optimization of charging infrastructure design and component sizing of a plug-in series HEV powertrain. In IFAC World Congress, Milano, Italy, 2011.

[21] N. Murgovski, L. Johannesson, J. Sjöberg, and B. Egardt. Component sizing of a plug-in hybrid electric powertrain via convex optimization. Mechatronics, 22(1):106-120, 2012.

[22] International Energy Agency. Technology roadmap: electric and plug-in hybrid electric vehicles. Technical report, IEA, Paris, France, 2011.

[23] X. Wu, B. Cao, X. Li, J. Xu, and X. Ren. Component sizing optimization of plug-in hybrid electric vehicles. Applied Energy, 88(3):799-804, March 2011.

[24] S. Boyd and L. Vandenberghe. Convex Optimization. Cambridge University Press, 2004. 
[25] C.S.N. Shiau, N. Kaushal, C.T. Hendrickson, S.B. Peterson, J.F. Whitacre, and J.J. Michalek. Optimal plug-in hybrid electric vehicle design and allocation for minimum life cycle cost, petroleum consumption, and greenhouse gas emissions. Journal of Mechanical Design, 132:1-11, 2010.

[26] M. Montazeri-Gh and A. Poursamad. Application of genetic algorithm for simultaneous optimisation of hev component sizing and control strategy. International Journal of Alternative Propulsion, 1(1):63-78, 2006.

[27] L. Wu, Y. Wang, X. Yuan, and Z. Chen. Multiobjective optimization of HEV fuel economy and emissions using the self-adaptive fifferential evolution algorithm. IEEE Transactions on Vehicular Technology, 60(6):2458-2470, July 2011.

[28] J.F. Sturm. Using SeDuMi 1.02, a Matlab toolbox for optimization over symmetric cones. Optimization Methods and Software, 11(1-4):635-653, 1999.

[29] K.C. Toh, M.J. Todd, and R.H. Tütüncü. SDPT3 - a Matlab software package for semidefinite programming. Optimization Methods and Software, 11:545-581, 1999.

[30] CVX Research. CVX: Matlab software for disciplined convex programming, version 2.0 beta. Www.cvxr.com/cvx, November 2013.

[31] M. Grant and S. Boyd. Graph implementations for nonsmooth convex programs. In V. Blondel, S. Boyd, and H. Kimura, editors, Recent Advances in Learning and Control, Lecture Notes in Control and Information Sciences, pages 95-110. Springer-Verlag Limited, 2008.

[32] J. Löfberg. YALMIP : A toolbox for modeling and optimization in Matlab. In Proceedings of the CACSD Conference, Taipei, Taiwan, 2004.

[33] Saft. Available online: www.saftbatteries.com, November 2013.

[34] Maxwell. Available online: www.maxwell.com, November 2013.

[35] N. Murgovski, L. Johannesson, A. Grauers, and J. Sjöberg. Dimensioning and control of a thermally constrained double buffer plug-in HEV powertrain. In 51st IEEE Conference on Decision and Control, Maui, Hawaii, December 10-13 2012.

[36] N. Murgovski, L. Johannesson, and J. Sjöberg. Engine on/off control for dimensioning hybrid electric powertrains via convex optimization. IEEE Transactions on Vehicular Technology, 62(7):2949-2962, 2013.

[37] M. Pourabdollah, N. Murgovski, A. Grauers, and B. Egardt. Optimal sizing of a parallel 
PHEV powertrain. IEEE Transactions on Vehicular Technology, 62(6):2469-2480, 2013.

[38] N. Murgovski, L. Johannesson, and B. Egardt. Optimal battery dimensioning and control of a CVT PHEV powertrain. IEEE Transactions on Vehicular Technology, 2013. Accepted for publication.

[39] S. Ebbesen, P. Elbert, and L. Guzzella. Battery state-of-health perceptive energy management for hybrid electric vehicles. IEEE Transactions On Vehicular Technology, 61(7):2893$2900,2012$.

[40] L. Johannesson, N. Murgovski, S. Ebbessen, B. Egardt, E. Gelso, and J.Hellgren. Including a battery state of health model in the HEV component sizing and optimal control problem. In IFAC Advances in Automotive Control (AAC), Tokyo, Japan, 2013. 


\section{Sidebar 1: Hybrid Electric Powertrains}

There are many ways to configure a hybrid electric powertrain. For the discussions in this article, we will focus on two common and relatively simple configurations, namely the parallel and the series hybrid powertrains. The principal layout of a simple hybrid electric powertrain with a parallel configuration is shown in Figure S1. In this case, the two components providing the motive force, the internal combustion engine (ICE) and the electric machine (EM), are both mechanically coupled to the drive shaft of the vehicle. The total torque delivered is the sum of the individual torques, providing an additional degree of freedom in the operation of the vehicle compared to a conventional vehicle. In addition, the parallel hybrid powertrain offers the possibility to run purely electrically in zero emission areas. In this case, the electric machine works as a motor, and the combustion engine is decoupled from the driveline by a clutch. The electric machine can also be operated as a generator in order to recuperate braking energy. Depending on the operating mode of the powertrain, the energy buffer, usually a battery, is charged or discharged.

In contrast to the parallel configuration, a series powertrain is characterized by the absence of a mechanical connection between the internal combustion engine and the wheels, as illustrated in Figure S2. Instead, the wheels are driven entirely by an electric machine without the need for a transmission. The electric machine obtains electricity either from a generator, coupled to the ICE, or the buffer. This powertrain gives a choice of the ICE's speed and torque, regardless of the vehicle speed. Thus, the engine can be operated at torque-speed points that minimize emissions and combined losses of the ICE and the generator, [4]. For this reason, these two components can be considered as a single unit, an engine-generator unit (EGU). 


\section{Sidebar 2: Convex Optimization}

A convex optimization problem in standard form is defined as follows

$$
\begin{aligned}
\operatorname{minimize} & f(x) \\
\text { subject to } & g_{i}(x) \leq 0, \quad i=1, \ldots, m \\
& A x=b,
\end{aligned}
$$

where $x=\left(x_{1}, \ldots, x_{n}\right)$ are the optimization variables, and it is required that the objective function $f$ and the constraint functions $\left\{g_{i}\right\}$ are convex, that is,

$$
f(\theta x+(1-\theta) y) \leq \theta f(x)+(1-\theta) f(y), \quad 0 \leq \theta \leq 1
$$

See Figure S3 for an illustration.

A convex optimization problem has two important properties that make it computationally attractive. First, the feasible set, that is the set of all $x$ that fulfill the constraints, is convex; this means that for any two points $x, y$ in the set the line segment between also lies in the set. Second, the optimization problem has a unique global optimum. These characteristics of the problem allow the construction of efficient numerical algorithms, implying that even very large problems can be solved with moderate computational resources and time. A useful reference for further reading is [24]. 


\section{Sidebar 3: Constraints Relaxation}

The relaxation of the dissipation functions to inequalities is an important step to transform the optimization problem into standard convex form. It can be understood intuitively that the relaxation will not change the properties of the optimal solution, but a more rigorous argument can also be provided, as will be shown. By defining the functions

$$
\begin{aligned}
& g_{G}\left(\boldsymbol{P}_{G e}, s_{G}\right)=\boldsymbol{\sigma}_{G} s_{G} f_{G}\left(\frac{\boldsymbol{P}_{G e}}{s_{G}}\right)+\boldsymbol{P}_{G e}, \\
& g_{B}\left(\boldsymbol{P}_{B t}, \boldsymbol{E}_{B}, s_{B}\right)=s_{B} f_{B}\left(\frac{-\delta \boldsymbol{E}_{B}}{s_{B}}, \frac{\boldsymbol{E}_{B}}{s_{B}}\right)+\boldsymbol{P}_{B t}, \\
& g_{M}\left(\boldsymbol{P}_{M m}, s_{M}\right)=s_{M} f_{M}^{\omega_{M}}\left(\frac{\boldsymbol{P}_{M m}}{s_{M}}\right)+\boldsymbol{P}_{M m}, \\
& g_{T}\left(\boldsymbol{P}_{T 2}, s_{T}\right)=s_{T} f_{T}^{\boldsymbol{\omega}_{T}}\left(\frac{\boldsymbol{P}_{T 2}}{s_{T}}\right)+\boldsymbol{P}_{T 2},
\end{aligned}
$$

the optimization problem from (40)-(44) can be written as

$$
\begin{array}{cl}
\text { minimize } & c=\rho_{e} \Delta t \mathbf{1}^{T} \boldsymbol{P}_{e}+\rho_{f} \Delta t \mathbf{1}^{T} g_{G}\left(\boldsymbol{P}_{G e}, s_{G}\right)+\sum_{X} c_{X}\left(s_{X}\right), \\
\text { subject to } & \boldsymbol{P}_{e} \geq g_{B}\left(\boldsymbol{P}_{B t}, \boldsymbol{E}_{B}, s_{B}\right)+\delta \boldsymbol{E}_{B}, \\
& \boldsymbol{P}_{B t}+\boldsymbol{P}_{G e} \geq g_{M}\left(\boldsymbol{P}_{M m}, s_{M}\right)+h_{M}\left(s_{M}\right), \\
& \boldsymbol{P}_{M m} \geq g_{T}\left(\boldsymbol{P}_{T 2}, s_{T}\right)+h_{T}\left(s_{T}\right), \\
& \boldsymbol{P}_{T 2} \geq h(s)
\end{array}
$$

where $h_{M}, h_{T}, h$ are functions of the scaling parameters only, and constraints from component limitations and vehicle requirements have been omitted to simplify the arguments (this implies, in particular, that no mechanical braking power is needed). The functions $\left\{g_{X}\right\}$ are convex by construction and are required to be strictly increasing in their first arguments. In terms of the generic component model in Figure 3, this means that it is required that $\frac{\partial P_{1}}{\partial P_{2}}>0$, that is, the incremental input-output (or vice versa) gain is positive, which is a natural and mild condition. An illustration of the function $g_{M}\left(\boldsymbol{P}_{M m}, s_{M}\right)$ is provided in Figure S4.

Consider now an optimal solution, with optimal values denoted by *, for which the relaxed 
constraints can be written as

$$
\begin{aligned}
& \boldsymbol{P}_{e}^{*}=g_{B}\left(\boldsymbol{P}_{B t}^{*}, \boldsymbol{E}_{B}^{*}, s_{B}^{*}\right)+\delta \boldsymbol{E}_{B}^{*}+\gamma_{1}, \\
& \boldsymbol{P}_{B t}^{*}+\boldsymbol{P}_{G e}^{*}=g_{M}\left(\boldsymbol{P}_{M m}^{*}, s_{M}^{*}\right)+h_{M}\left(s_{M}^{*}\right)+\gamma_{2}, \\
& \boldsymbol{P}_{M m}^{*}=g_{T}\left(\boldsymbol{P}_{T 2}^{*}, s_{T}^{*}\right)+h_{T}\left(s_{T}^{*}\right)+\gamma_{3}, \\
& \boldsymbol{P}_{T 2}^{*}=h\left(s^{*}\right)+\gamma_{4},
\end{aligned}
$$

where $\gamma_{j}, j=1, \ldots, 4$ are nonnegative slack variables. Now, define a suboptimal feasible solution from $\boldsymbol{P}_{B t}^{*}, \boldsymbol{E}_{B}^{*}, s^{*}$ and the power variables $\tilde{\boldsymbol{P}}_{e}, \tilde{\boldsymbol{P}}_{G e}, \tilde{\boldsymbol{P}}_{M m}, \tilde{\boldsymbol{P}}_{T 2}$, shifted from the optimal by

$$
\begin{aligned}
& \tilde{\boldsymbol{P}}_{T 2}=\boldsymbol{P}_{T 2}^{*}-\gamma_{4} \leq \boldsymbol{P}_{T 2}^{*}, \\
& \tilde{\boldsymbol{P}}_{M m}=\boldsymbol{P}_{M m}^{*}-\gamma_{3}-\Delta g_{T} \leq \boldsymbol{P}_{M m}^{*}, \\
& \tilde{\boldsymbol{P}}_{G e}=\boldsymbol{P}_{G e}^{*}-\gamma_{2}-\Delta g_{M} \leq \boldsymbol{P}_{G e}^{*} \\
& \tilde{\boldsymbol{P}}_{e}=\boldsymbol{P}_{e}^{*}-\gamma_{1} \leq \boldsymbol{P}_{e}^{*}
\end{aligned}
$$

where the non-negativeness of the vectors

$$
\begin{aligned}
& \Delta g_{T}=g_{T}\left(\boldsymbol{P}_{T 2}^{*}, s_{T}^{*}\right)-g_{T}\left(\tilde{\boldsymbol{P}}_{T 2}, s_{T}^{*}\right), \\
& \Delta g_{M}=g_{M}\left(\boldsymbol{P}_{M m}^{*}, s_{M}^{*}\right)-g_{M}\left(\tilde{\boldsymbol{P}}_{M m}, s_{M}^{*}\right), \\
& \Delta g_{G}=g_{G}\left(\boldsymbol{P}_{G e}^{*}, s_{G}^{*}\right)-g_{G}\left(\tilde{\boldsymbol{P}}_{G e}, s_{G}^{*}\right),
\end{aligned}
$$

follows from the monotonicity assumption. The cost of the suboptimal solution can now be related to the optimal solution as follows

$$
\begin{aligned}
\tilde{c} & =\rho_{e} \Delta t \mathbf{1}^{T} \tilde{\boldsymbol{P}}_{e}+\rho_{f} \Delta t \mathbf{1}^{T} g_{G}\left(\tilde{\boldsymbol{P}}_{G e}, s_{G}^{*}\right)+\sum_{X} c_{X}\left(s_{X}^{*}\right) \\
& =c^{*}-\rho_{e} \Delta t \mathbf{1}^{T} \gamma_{1}-\rho_{f} \Delta t \mathbf{1}^{T} \Delta g_{G} \leq c^{*}
\end{aligned}
$$

Since $c^{*}$ is the optimal cost, it follows that $\gamma_{1}=0$ and $\Delta g_{G}=0$, so that $\tilde{\boldsymbol{P}}_{e}=\boldsymbol{P}_{e}^{*}$ and $\tilde{\boldsymbol{P}}_{G e}=\boldsymbol{P}_{G e}^{*}$. From the non-negativeness of the $\Delta g$ vectors it follows that the other variables are also equal, all slack variables are 0 , and the optimal solution fulfils the constraints with equality. The argument can be carried out for the case with component constraints, but the notation becomes more involved. 


\section{Author Information}

Bo Egardt (bo.egardt@chalmers.se) received the M.Sc. degree in electrical engineering and the Ph.D. degree in automatic control from Lund Institute of Technology, Sweden, in 1974 and 1979, respectively. During 1980, he was a Research Associate at the Information Systems Laboratory, Stanford, CA. From 1981 to 1989, he was with Asea Brown Boveri, where he was heavily involved in the introduction of adaptive control in the process industry. In 1989, he was appointed Professor of automatic control at Chalmers University of Technology, Sweden. His main areas of interest include adaptive and hybrid control and applications of control in the automotive area. He has been an Associate Editor of IEEE Transactions on Control Systems Technology and of the European Journal of Control. He is a member of the editorial board for the International Journal of Adaptive Control and Signal Processing. He is a Fellow of the IEEE.

Nikolce Murgovski received the M.Sc. degree in Software Engineering from University West, Sweden, 2007, the M.Sc. degree in Applied Physics, and the Ph.D. degree in Signals and Systems from Chalmers University of Technology, Sweden, in 2007 and 2012, respectively. Currently he is a Post-Doctoral Researcher at the Department of Signals and Systems at Chalmers University. His research activities include optimal control and dimensioning of automotive powertrains.

Mitra Pourabdollah received the B.Sc. degree in electrical engineering from Shiraz University, Iran, in 2006, and the M.Sc. degree in systems, control and robotics from Royal University of Technology, Sweden, in 2009. In 2009 she joined the automatic control group at Chalmers University of Technology as a Ph.D. student. Her research topics include energy management and optimal sizing of automotive powertrains.

Lars Johannesson Mårdh received the M.Sc. degree in Automation and Mechatronics and the Ph.D. degree in automatic control from Chalmers University of Technology, Sweden in 2004 and 2009, respectively. He has been with the Electromobility group at Viktoria Swedish ICT since 2011, working with research on powertrain control within the Chalmers Energy Initiative. His main research interests are optimal control of hybrid and plug-in hybrid electric vehicles, control of auxiliary systems in trucks, active cell balancing, and system studies of hybrid vehicles. He is a member of the IEEE. 


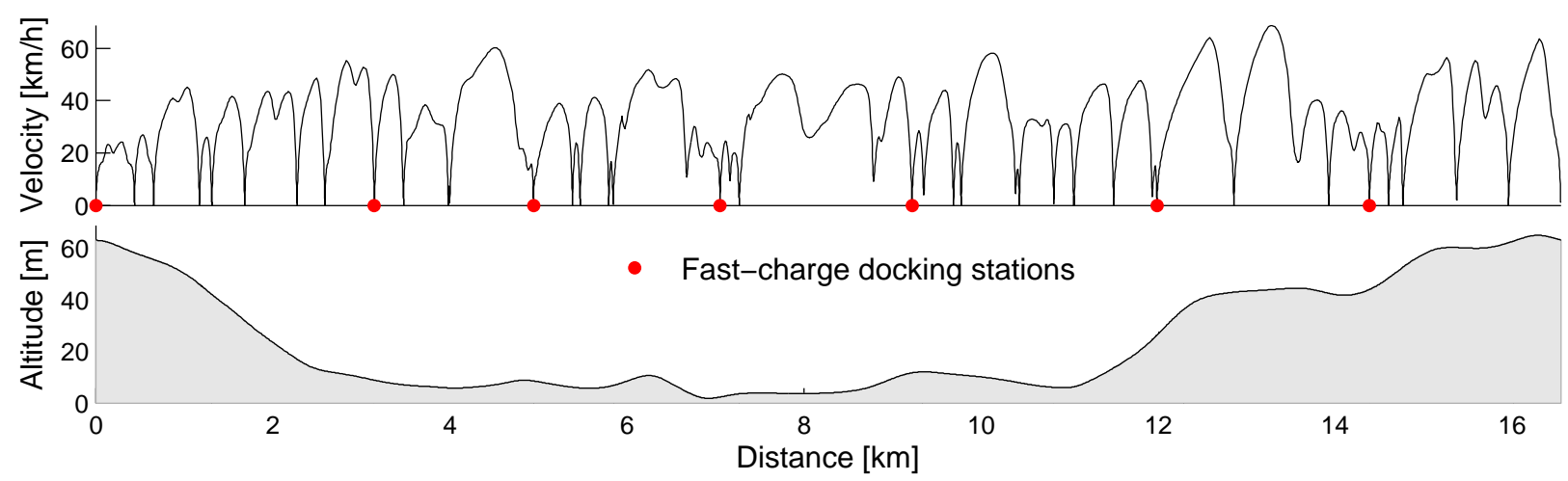

Figure 1. Bus line described by demanded velocity and road altitude. The line is circular, that is, it starts and ends at the same bus stop. The bus line is equipped with fast-charge docking stations installed on seven bus stops. 


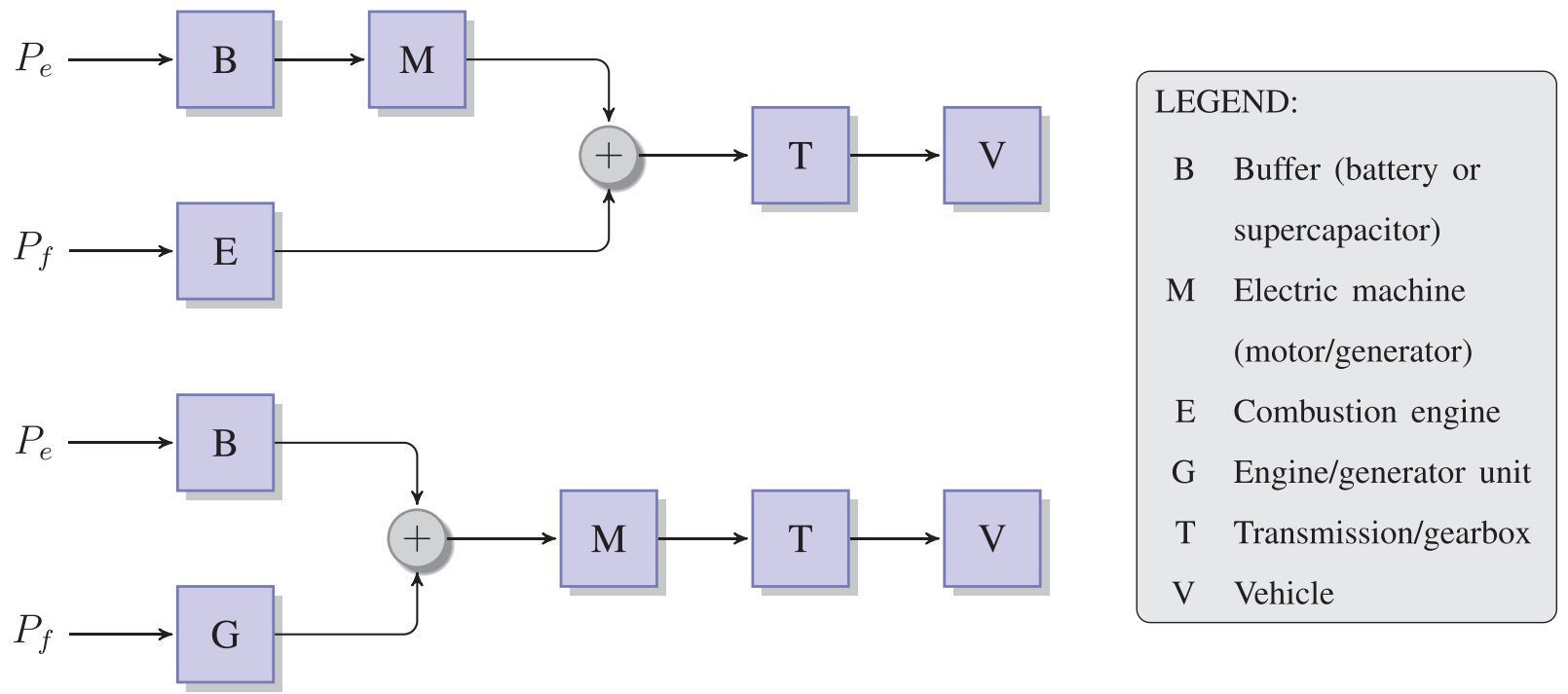

Figure 2. Power flows in parallel (above) and series (below) powertrains. The arbitration between the two power sources is represented by the junction of two mechanical (parallel) and electric (series) power flows, respectively. The primary power sources are fuel power $P_{f}$ and electric power $P_{e}$, the latter representing grid connection for a plug-in hybrid. 


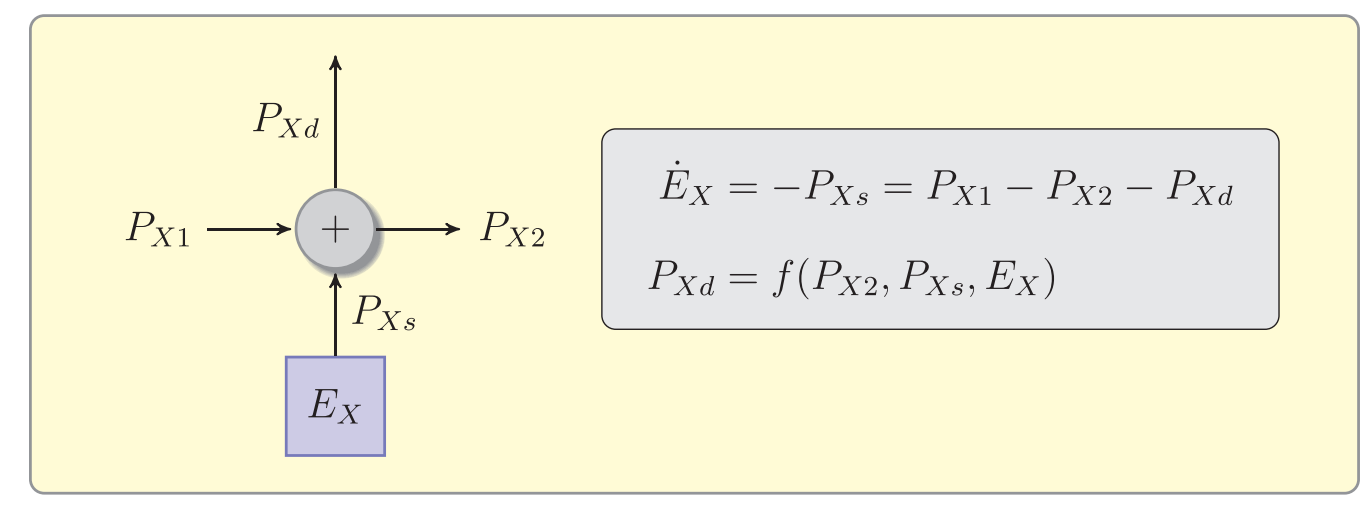

Figure 3. Generic component model. The model expresses a power flow balance, including a dissipation term $P_{X d}$ and a storage term $P_{X s}$. The dissipation function $f$ is assumed to be convex. 


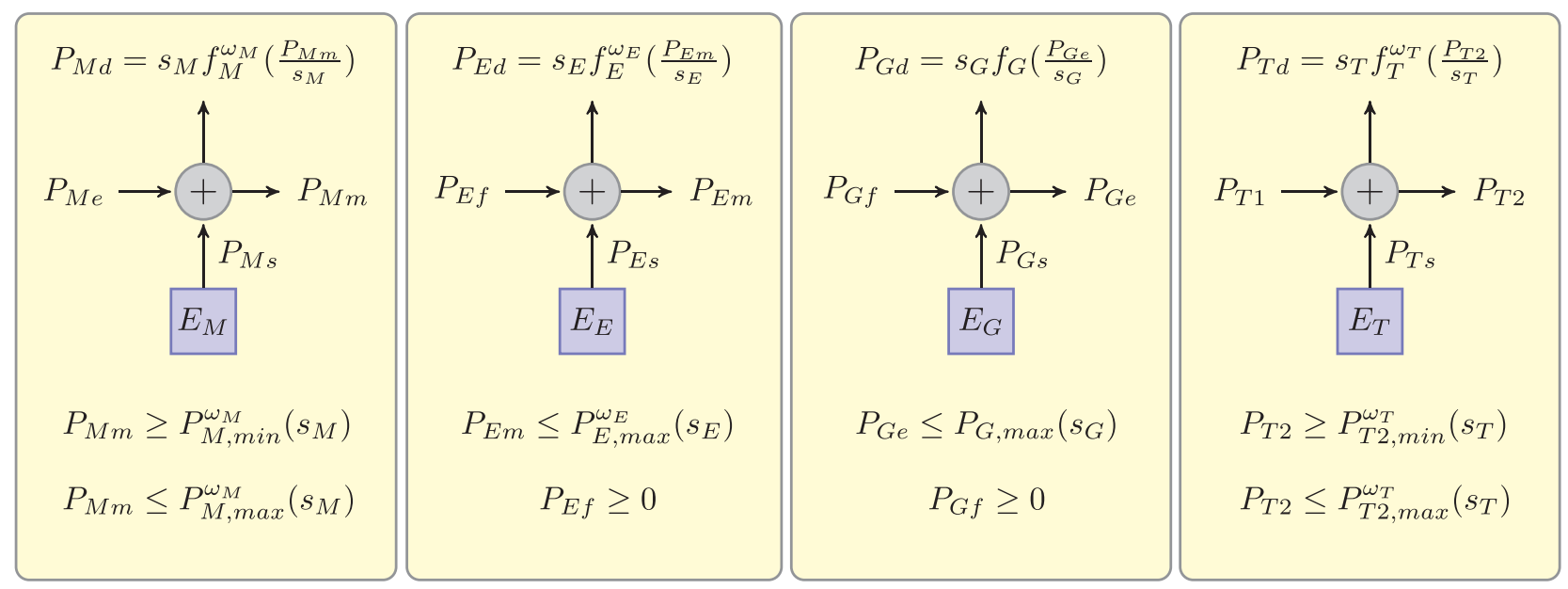

Figure 4. Models for the rotating machines. The figure summarizes the notations used for, left to right, electrical motor (M), combustion engine (E), engine/generator unit $(\mathrm{G})$, and transmission/gearbox (T). Subscripts used are $f$ for fuel, $e$ for electrical, and $m$ for mechanical. 


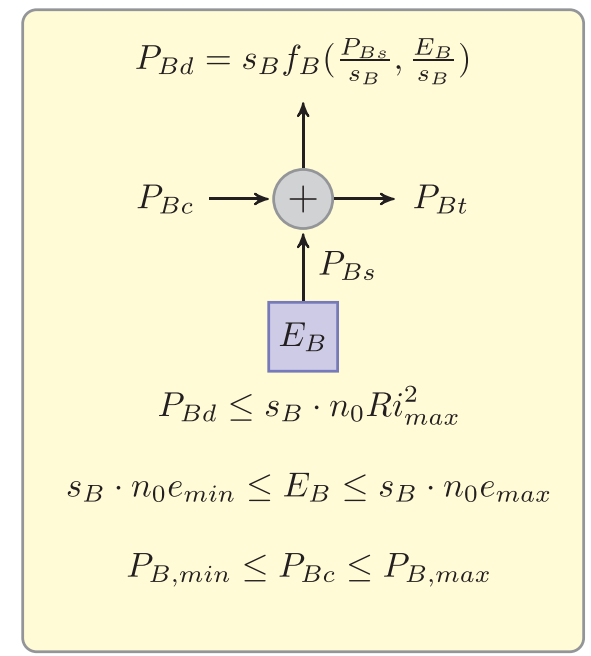

Figure 5. Energy buffer model. Subscripts $c$ and $t$ indicate charging and terminal, respectively. 


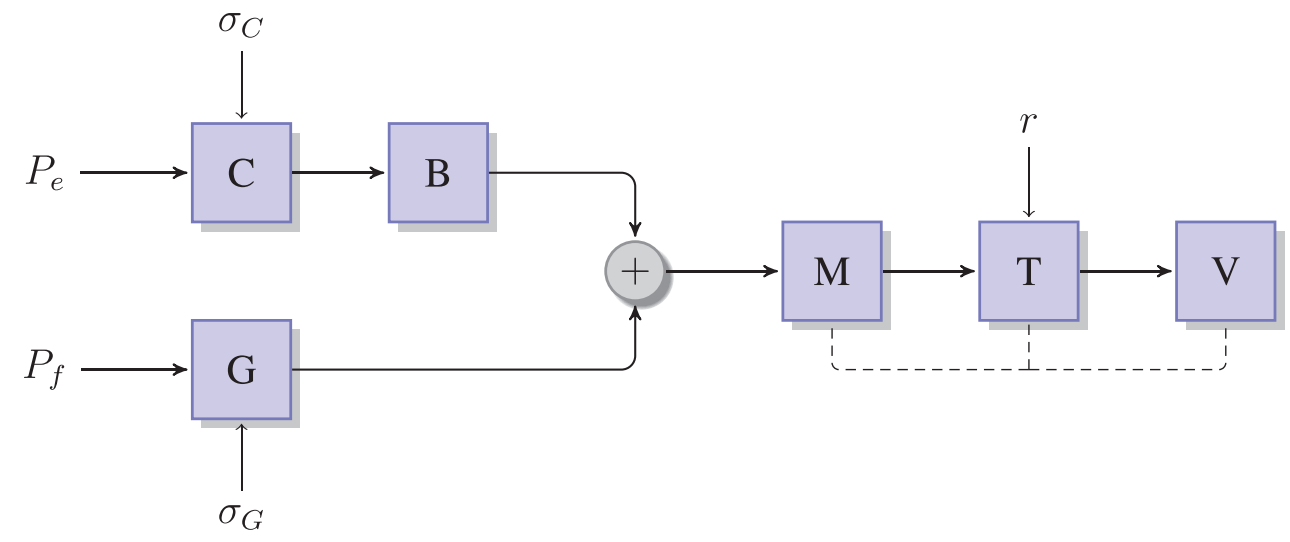

Figure 6. Model of the powertrain of a series plug-in vehicle. The component models are complemented by external logical signals, controlling gear switching as well as when charging from the grid is allowed, and when the engine/generator unit is turned on and off. 


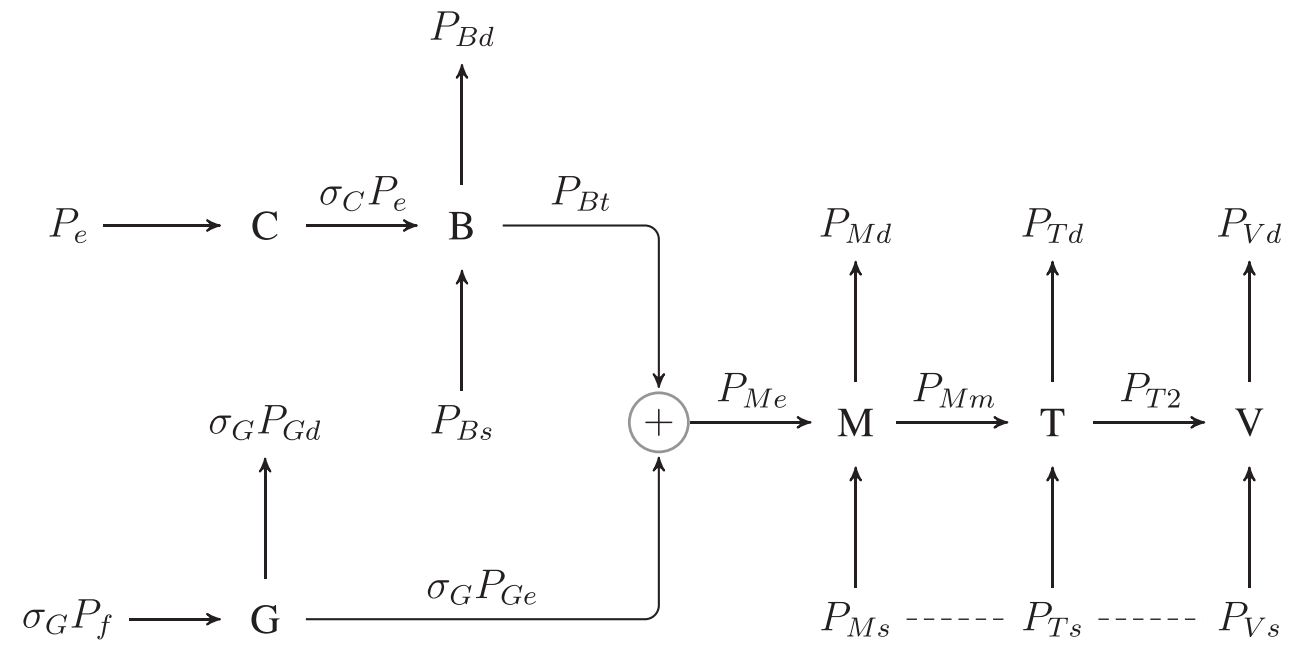

Figure 7. Model of the series PHEV, suitable for optimization. The figure is based on Figure 6 with component models added. The charger is assumed to be ideal, and the storage term of the engine/generator unit has been neglected. 


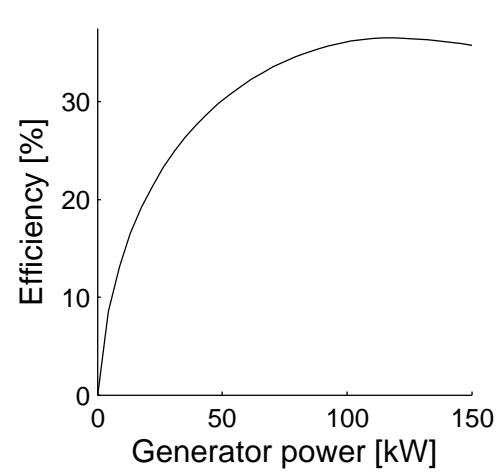

(a)

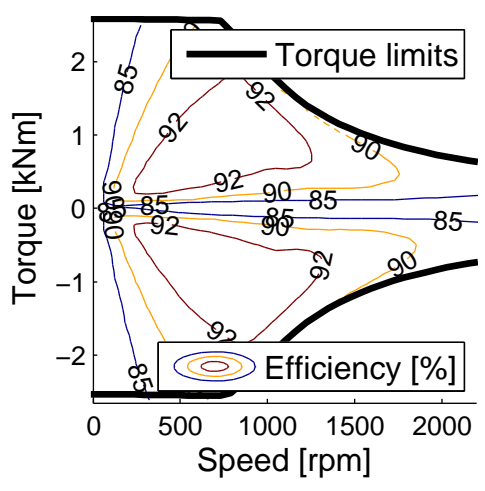

(b)

Figure 8. Model of engine generator unit (a) and electric machine (b). 


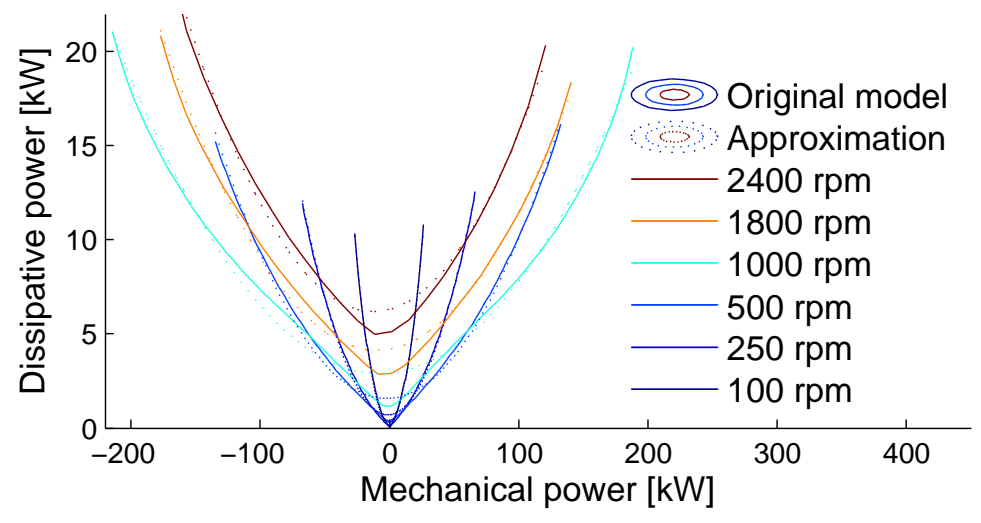

Figure 9. Electrical machine dissipative power vs. mechanical power at different speeds. The losses of the original model are depicted by the thick lines. The thin lines show quadratic approximation of the power losses. 


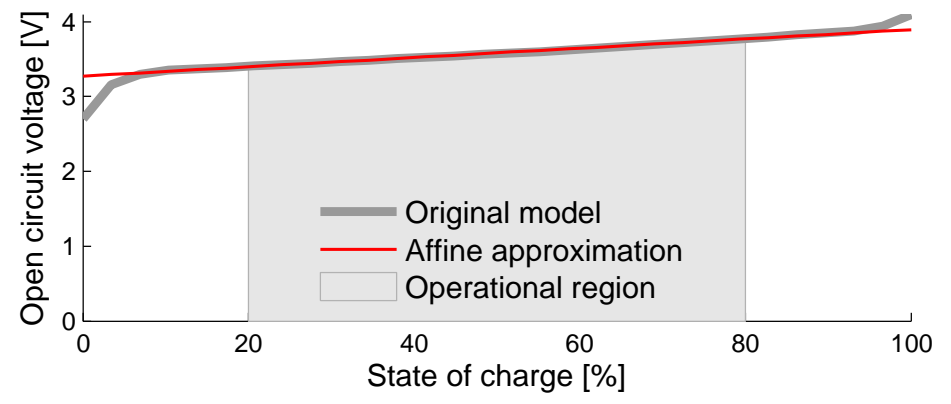

Figure 10. Battery cell open circuit voltage for the hybrid city bus considered in case study 1. The open circuit voltage is approximated as affine function in state-of-charge (SOC). In order to prolong battery life, the operational SOC range is restricted to be between $20 \%$ and $80 \%$. 

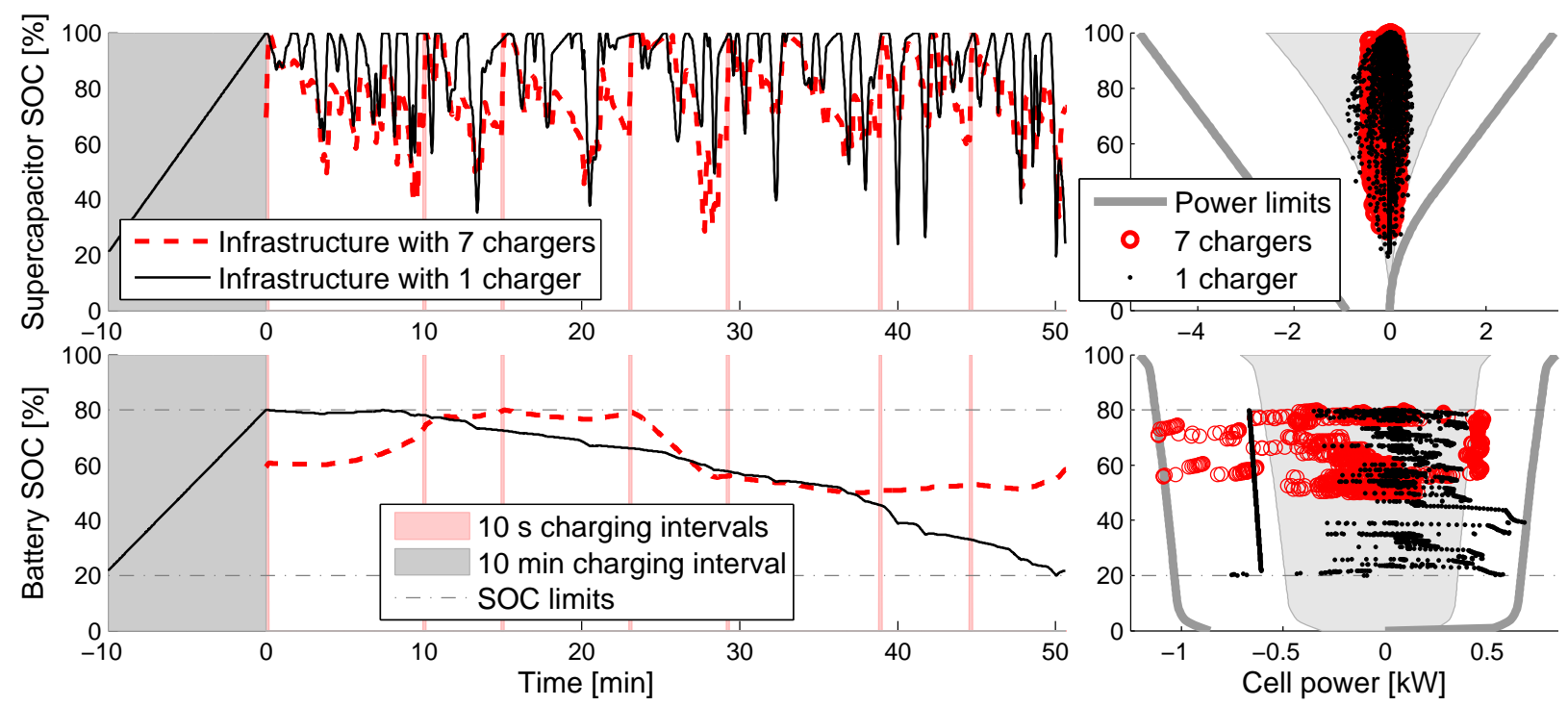

Figure 11. Optimal results for case study 1 for two charging scenarios. The first row shows results for the supercapacitor, and the second row for the battery. The plots to the left show the optimal SOC trajectories vs. time, while the plots to the right show the optimal SOC points vs. cell power. The shaded region in the right plots correspond to cell efficiency above $90 \%$. 


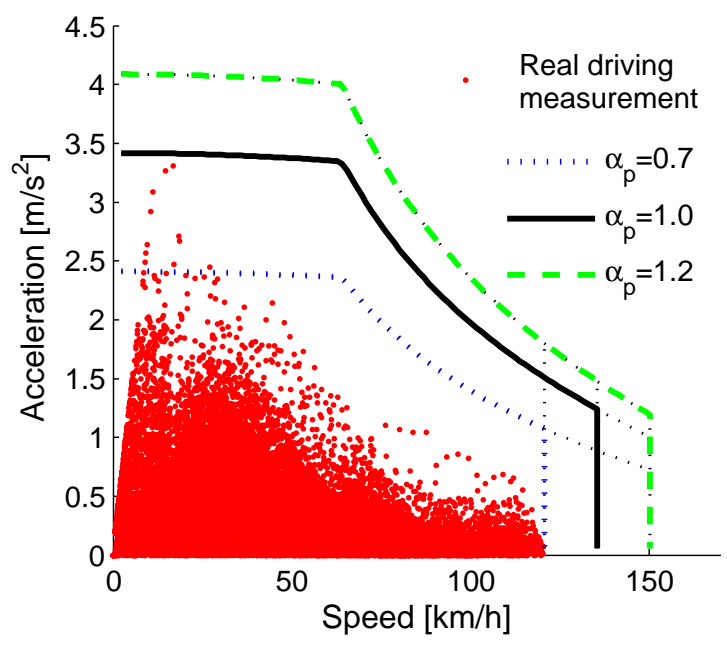

(a)

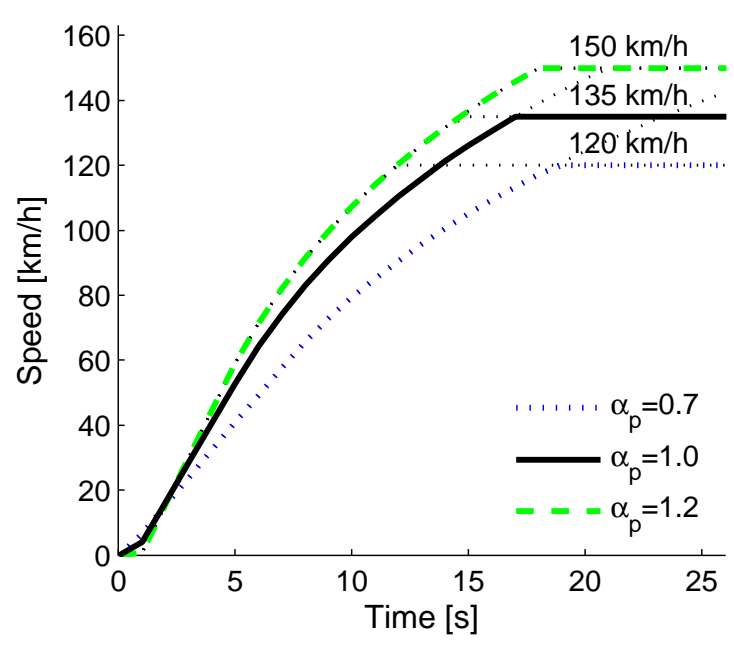

(b)

Figure 12. Examples of acceleration and top speed requirements (a) and corresponding performance cycles (b), appended to the real-life driving cycle. The different levels of performance requirements are obtained by multiplying the baseline curve by $\alpha_{p}$ and changing the top speed. The plot (a) also illustrates the operating points of the real-life driving cycle. 


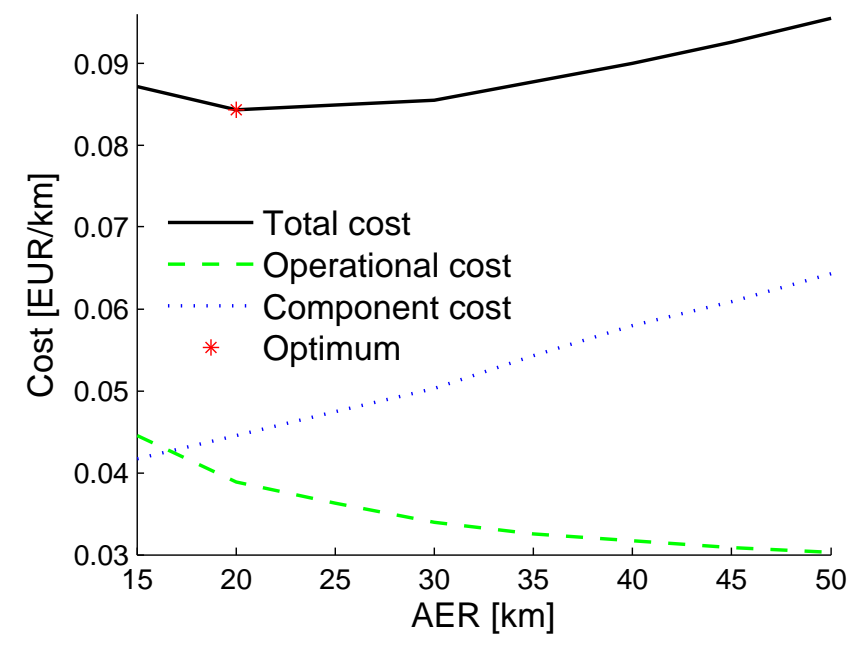

Figure 13. Optimal cost as function of all-electric-range (AER). The figure shows component, operational and total costs. The minimal total cost is shown with a star. 


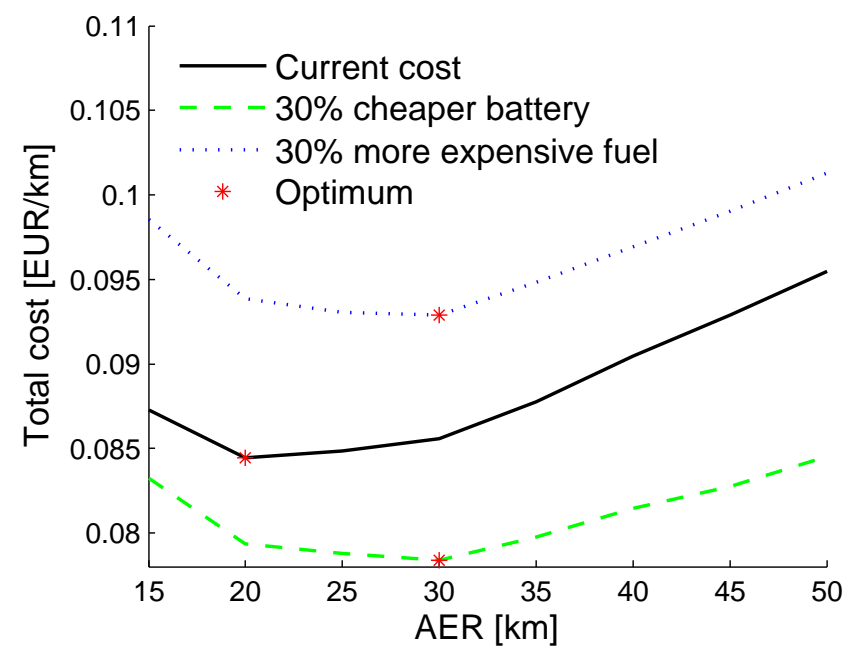

Figure 14. Optimal total cost as function of AER in different price scenarios. The scenarios are based on present battery and fuel cost, $30 \%$ cheaper batteries, and 30\% more expensive fuel. The minimal costs are shown with stars. 


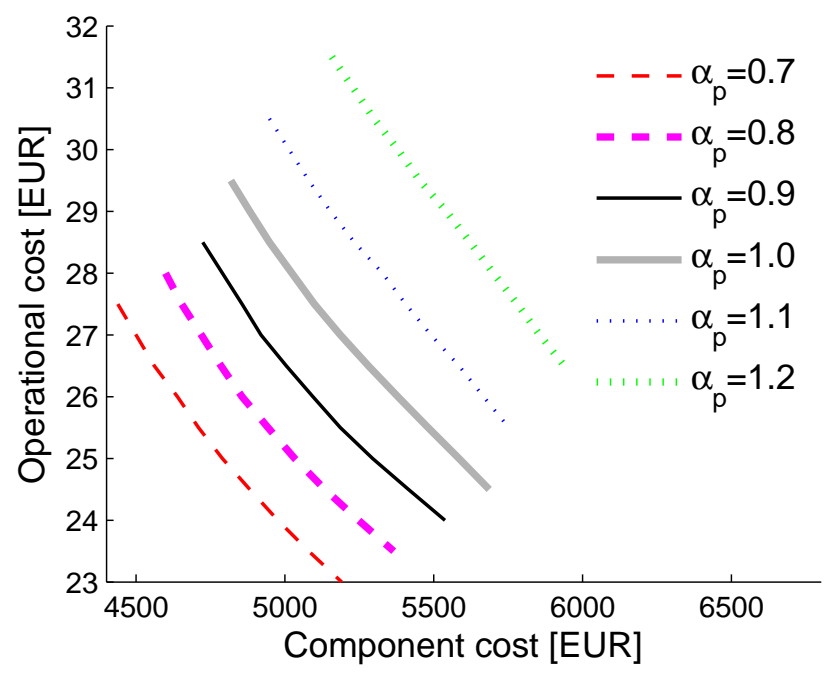

Figure 15. Set of Pareto points using different weighting factors between operational cost and component costs. 


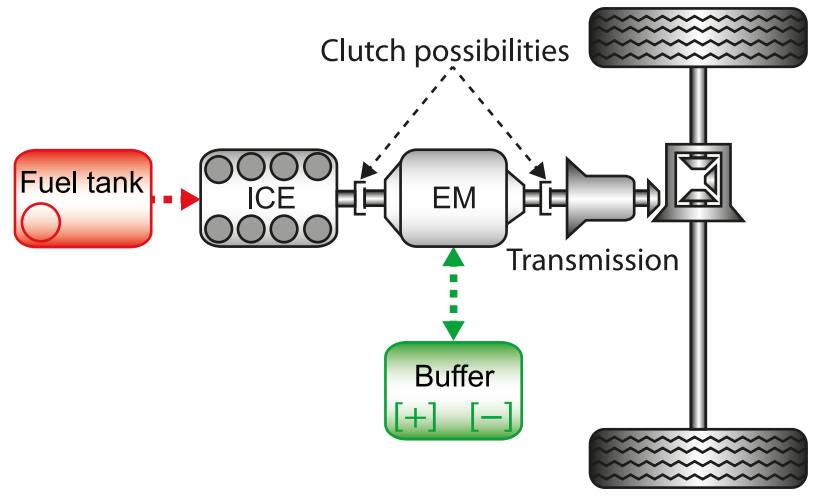

Figure S1. Parallel hybrid electric powertrain. The internal combustion engine (ICE) and the electric machine (EM) are both propelling the vehicle. The electric machine is connected to the battery (or supercapacitor), which acts as an energy buffer. The battery is discharged when the electric machine is used as motor and charged when the machine is used as a generator. 


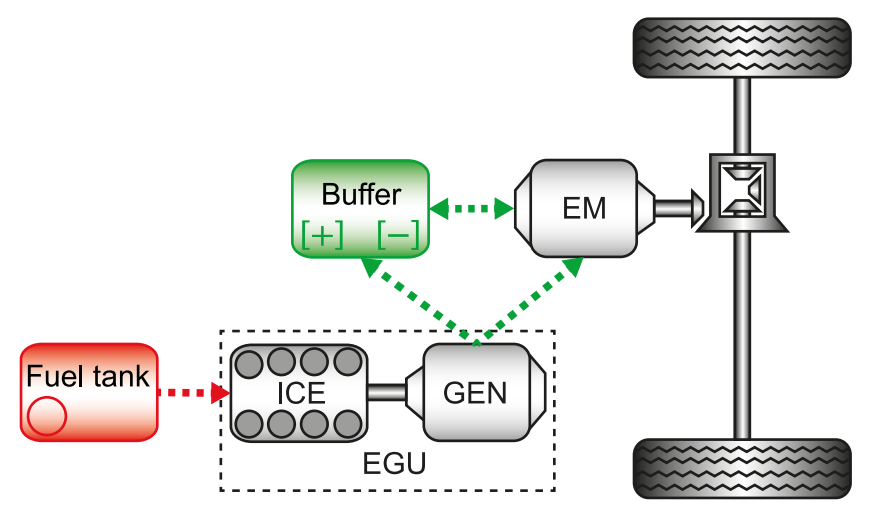

Figure S2. Series hybrid electric powertrain. The electric motor is propelling the vehicle. The battery is charged by the engine/generator unit (EGU), which is mechanically decoupled from the drive axle. 


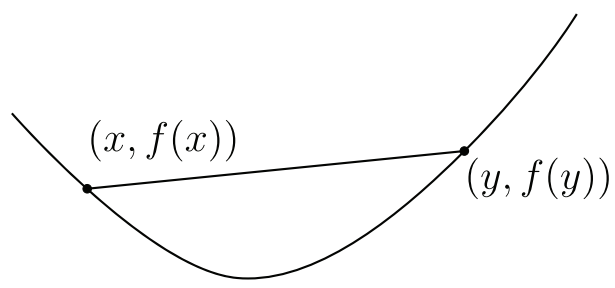

Figure S3. A convex function. The function graph is below the linear interpolation between any two points. 


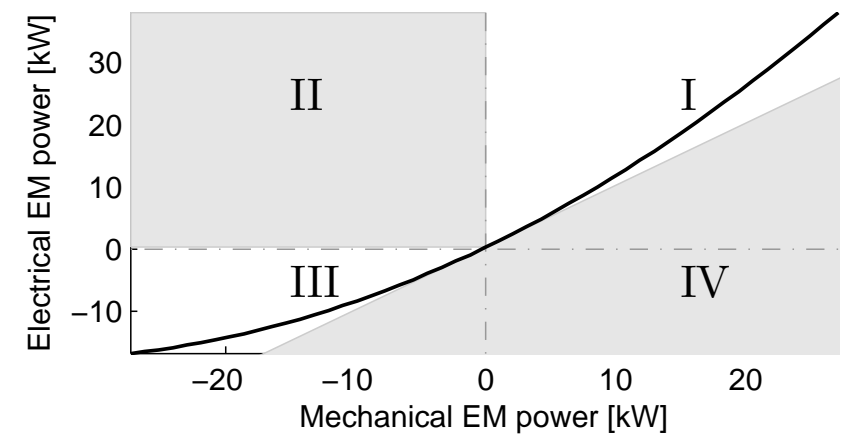

Figure S4. An example of a strictly increasing function illustrating electrical vs. mechanical EM power at $100 \mathrm{rpm}$. The function resides mainly in the first and third quadrant and it may pass through the second quadrant when friction losses (or idling losses) are present (zero torque and nonzero speed). The function can never reside in the shaded regions, where the upper region is defined by the second quadrant shifted upwards by the idling losses and the lower region is defined by the slope of unity passing through the idling losses. 